\title{
Assessment of Interface Trap Charges on Proposed TFET for Low Power High-frequency Application
}

\section{Sachin Kumar ( $\nabla$ sachin222sk@gmail.com )}

National Institute of Technology Hamirpur https://orcid.org/0000-0003-4827-4476

\section{Dharmendra Singh Yadav}

National Institute of Technology Hamirpur

\section{Research Article}

Keywords: SC-TFET, Analog/RF parameters, BTBT model, Linearity parameters

Posted Date: April 13th, 2021

DOI: https://doi.org/10.21203/rs.3.rs-286208/v1

License: (9) This work is licensed under a Creative Commons Attribution 4.0 International License. Read Full License

Version of Record: A version of this preprint was published at Silicon on January 25th, 2022. See the published version at https://doi.org/10.1007/s12633-021-01616-0. 


\title{
Assessment of Interface Trap Charges on Proposed TFET for Low Power High-Frequency Application
}

\author{
Sachin Kumar • Dharmendra Singh Yadav
}

Received: date / Accepted: date

\begin{abstract}
Accumulation of trap charges at the semiconductor and oxide interface is the most dominating factor and cannot be neglected as it degrades device performance and reliability. This manuscript, presents detailed investigation to analyze the impact of interface trap charges (ITCs) on the performance parameters of the proposed device i.e., heterogeneous dielectric dual metal gate step channel TFET (HD DMG SC-TFET). The comparative study is conducted with dual metal gate step channel TEFT (DMG SC-TFET). The proposed device shows improved current carrying capability, suppressed ambipolar behaviour with steeper subthreshold swing. The purpose of this study to determine the ITCs impact on DC characteristics and analog/RF electrical performance parameters of the proposed device. It further observed that the proposed device exhibit superior performance due to dielectric engineering at oxide layer. Moreover, advanced communication devices must respond linearly therefore, the impact of ITCs on linearity parameters is also studied. From this brief comparative investigation, it is observed that the proposed TFET exhibits negligible distortion in linearity parameters with little or no impact of trap charges as compared to DMG SC-TFET. Thus, proposed TFET is appropriate for ultra-low power high-frequency electronic devices.
\end{abstract}

Sachin Kumar

National Institute of Technology Hamirpur

Himachal Pradesh

Pin :- 177005, India

E-mail: sachin222sk@gmail.com

Dharmendra Singh Yadav

National Institute of Technology Hamirpur

Himachal Pradesh

Pin :- 177005, India

E-mail: dsyadav@nith.ac.in
Keywords SC-TFET • Analog/RF parameters . BTBT model $\cdot$ Linearity parameters

\section{Introduction}

The semiconductor industry shows rapidly growth in $20^{\text {th }}$ century. The reason behind this is MOSFETs. Nowadays, billions of transistors are inter-connect on single microprocessors for enhanced device performance. Today's transistors are the building block for low power electronic devices. So, MOSFETs is scaled down to increase device performance in term of switching speed, power consumption, and package density per unit area constant with reduced fabrication cost. This continuous down-scaling of MOSFETs has also lead to adverse impact on a device due to unacceptable enhancement in the leakages current that creates major problems at room temperature such as sub threshold leakage current, short circuit effect (SCE), mobility degradation, drain induced barrier lowing (DIBL), impact ionization, hot carrier effects (HCE) and static power consumption [1-3]. In addition to this MOSFET limits the subthreshold slope to $60 \mathrm{mV} /$ decades [4].

In this concern, the tunnel field-effect transistor (TFET) shows very promising behavior to overcomes the aforementioned limitation of MOSFET [5]. It offers sharper turn-on devices compared to MOSFET and shows better performance for low-power electronic device. TFET works on phenomena of quantum tunneling of charge carrier from source to channel which benefits steeper switching characteristics and reduced power consumption [6]. Apart from these advantages it also suffers from several limitations such as low current driving capability and ambipolar current behavior that degrades the 


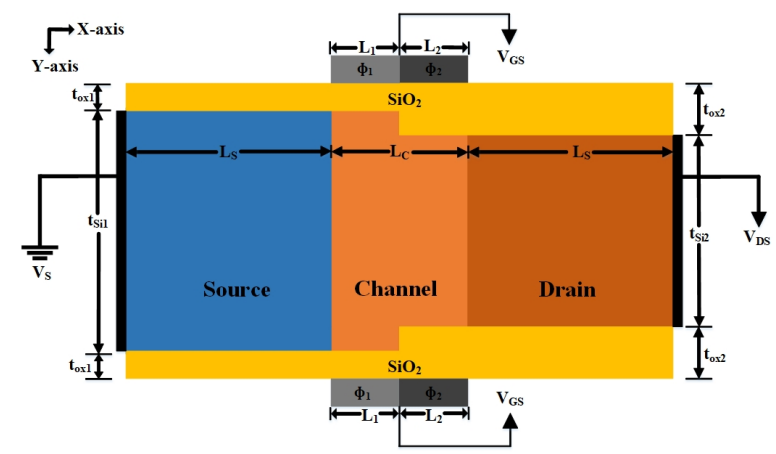

(a)

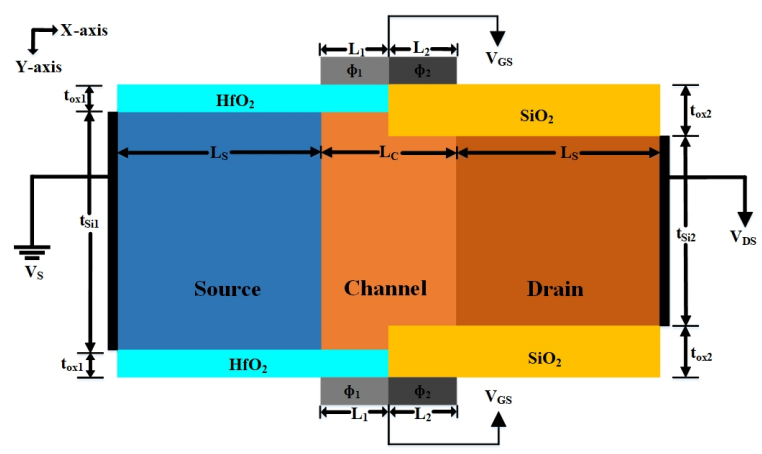

(b)

Fig. 1: Device Schematic view of (a) DMG SC-TEFT (b) HD DMG SC-TEFT

device analog/RF performance [7]. In order to overcome these limitations of conventional TFET various techniques are introduced such as gate engineering [8], dielectric engineering [9], doping engineering [10], material engineering [11] and many more.

In order to solve issues related to conventional TFET a novel combination of gate engineering with step channel structural modification [12] are implemented in this manuscript as dual metal gate step channel TFET (DMG SC-TFET). Due to this an intense improved ON-state current with steeper subthreshold swing is noticed. For further reducing threshold voltage and increase current driving capability implementation of dielectric engineering followed with gate engineering and step channel structural modification leads to our proposed device heterogeneous dielectric dual metal gate step channel TFET (HD DMG SC-TFET).

However, during fabrication process ITCs are generally accumulated at semiconductor/oxide interface due to damage induced by bond breaking, oxidation, radiation, and stress which are responsible for degrading the life span of the devices $[13,14]$. These traps charges are categorized as donor trap charges and acceptor trap charges. Donor trap charges are presented above the valance energy band which acts as a positive ITCs when it denotes an electron whereas acceptor trap charges are present below the conduction energy band and it acts as a negative ITCs when it accepts an electron $[15,16]$.

In TFETs these trap charges are accumulated because of missive electric field at the tunneling junction which excited silicon body surface that leads to modification of the electric field at source/channel junction (SC-junction) [17]. In addition, the output current is exponentially dependent on the electric field at junction [18] that results in a change in the current-carrying capability of devices. So, it is mandatory to determine the impact of trap charges on performance parameters of device.
This manuscript is arranged into different sections as follows: Section 2 discussed device structure, simulation parameters, and device specification. Whereas, Section 3 investigates the impact of ITCs on physical characteristics, analog/RF and linearity performance parameters. Finally, Section 4 concludes the important finding of this brief study.

\section{Devices Structure}

Previously various research works have been conducted to show the impact of oxide thickness on $\mathrm{I}_{\text {on }}$ and subthreshold swing $[19,20]$. These works particularly conclude that reduced oxide thickness provides higher oxide capacitance with improves gate control capability which allows faster transition of charge carrier through tunnel barrier that results in enhance $\mathrm{I}_{\mathrm{on}}$ with stepper subthreshold swing [21]. However, to avoid high leakage current, oxide thickness is practically limited to not less than $1 \mathrm{~nm}$ [22]. Therefore to maintain lower leakage current that make a power efficient device we have made oxide layer of $2 \mathrm{~nm}$ thickness [23,24] at the drainchannel junction and to enhances Ion with steeper subthreshold swing the oxide thickness is kept as 1nm [25] at the source-channel junction that allows a greater number of fanouts with higher switching rate. This leads to step channel structure.

To overcome the challenges of conventional TFET, we have proposed a novel schematic of TFETs which are illustrated in Fig.1. Fig.1a and Fig.1b portrayed the device schematic cross-section view of DMG SCTFET and HD DMG SC-TFET. For DMG SC-TFET gate engineering is employed on the gate electrode with decreased oxide layer thickness at SC-junction. This allows better subthreshold swing and high current driving capability in the range $10^{-5}$ with suppressed ambipolar behavior in the range $10^{-15}$. For HD DMG SC-TFET oxide layer at SC-junction is deposited with hafnium 

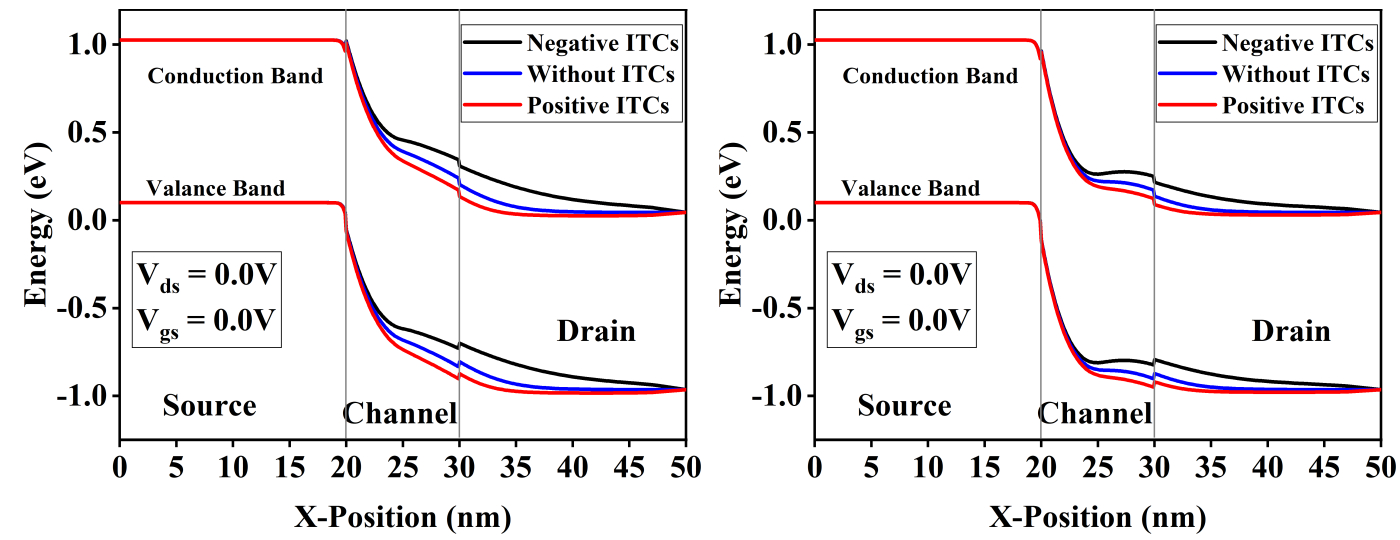

(a)

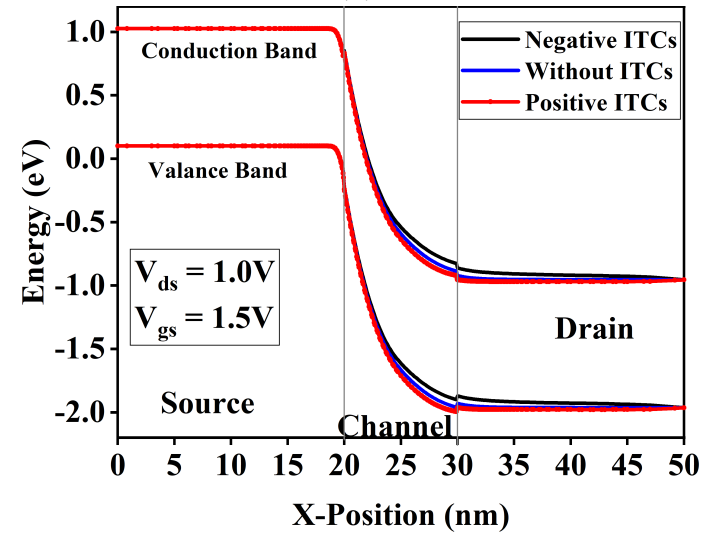

(c)

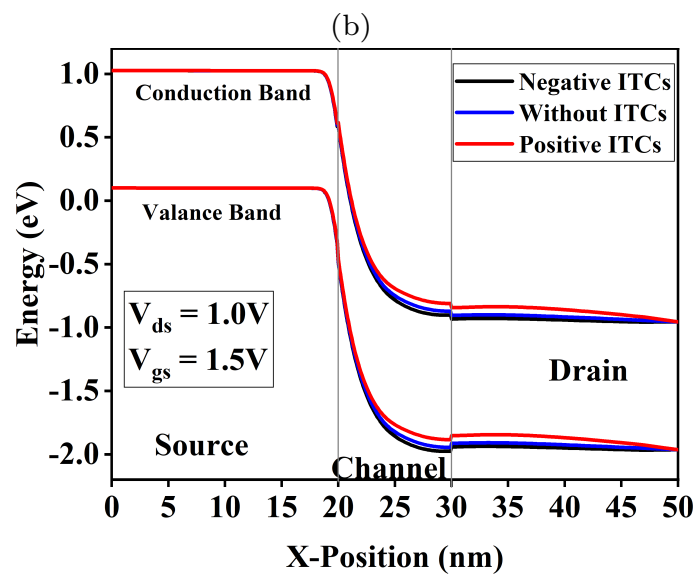

(d)

Fig. 2: Impact of ITC on (a) Energy Band of DMG SC-TFET under Thermal Equilibrium (b) Energy Band of HD DMG SC-TFET under Thermal Equilibrium (c) Energy Band of DMG SC-TFET under ON-State (d) Energy Band of HD DMG SC-TFET under ON-State

Table 1: Structural parameters value

\begin{tabular}{ll}
\hline Parameters (Notation) & Value \& Unit \\
\hline Source (p-type) doping $\left(\mathrm{N}_{\mathrm{S}}\right)$ & $5 \times 10^{20} \mathrm{~cm}^{-3}$ \\
Channel doping $\left(\mathrm{N}_{\mathrm{C}}\right)$ & $1 \times 10^{17} \mathrm{~cm}^{-3}$ \\
Drain (n-type) doping $\left(\mathrm{N}_{\mathrm{D}}\right)$ & $5 \times 10^{18} \mathrm{~cm}^{-3}$ \\
Source length $\left(\mathrm{L}_{\mathrm{S}}\right)$ & $20 \mathrm{~nm}$ \\
Oxide thickness at SC-junction $\left(\mathrm{t}_{\mathrm{ox} 1}\right)$ & $1 \mathrm{~nm}$ \\
Channel length $\left(\mathrm{L}_{\mathrm{C}}\right)$ & $10 \mathrm{~nm}$ \\
Oxide thickness at DC-junction $\left(\mathrm{t}_{\mathrm{ox} 2}\right)$ & $2 \mathrm{~nm}$ \\
Drain length $\left(\mathrm{L}_{\mathrm{D}}\right)$ & $20 \mathrm{~nm}$ \\
Source thickness $\left(\mathrm{t}_{\mathrm{si} 1}\right)$ & $8 \mathrm{~nm}$ \\
Drain thickness $\left(\mathrm{t}_{\mathrm{si} 2}\right)$ & $6 \mathrm{~nm}$ \\
Tunnel gate length $\left(\mathrm{L}_{1}\right)$ & $5 \mathrm{~nm}$ \\
Auxiliary gate length $\left(\mathrm{L}_{2}\right)$ & $5 \mathrm{~nm}$ \\
Tunnel gate work function $\left(\phi_{1}\right)$ & $4.02 \mathrm{eV}$ \\
Auxiliary gate work function $\left(\phi_{2}\right)$ & $4.50 \mathrm{eV}$ \\
\hline
\end{tabular}

oxide $\left(\mathrm{HfO}_{2}\right)$ that increases the tunneling probability of charge carrier at $\mathrm{SC}$-junction. This leads to a reduc- tion in threshold voltage and enhances current driving capability in the range of $10^{-4}$.

The simulation is carried out using TCAD software called Silvaco Atlas Simulator. Since, TFET is works on phenomena of quantum tunneling of charge carrier through potential barrier, bandgap narrowing (BGN) model and band to band tunneling (BTBT) models are used for simulation. In addition to these models some more physical models are used in this study such as Shockley read hall (SRH) model, concentration depended on mobility (CONMOB) model, field depended on mobility (FLDMOB) model, and Auger recombination (Auger) model. Also newton methods are employed for mathematical calculation. To investigates the impact of ITCs on electrical performance parameters of proposed TFET interfacial charge density are taken as $1 \times 10^{12} \mathrm{~cm}^{-3}$. Further, Table 1 depicted device specification and parameters that we used in the simulation of both TFETs. 


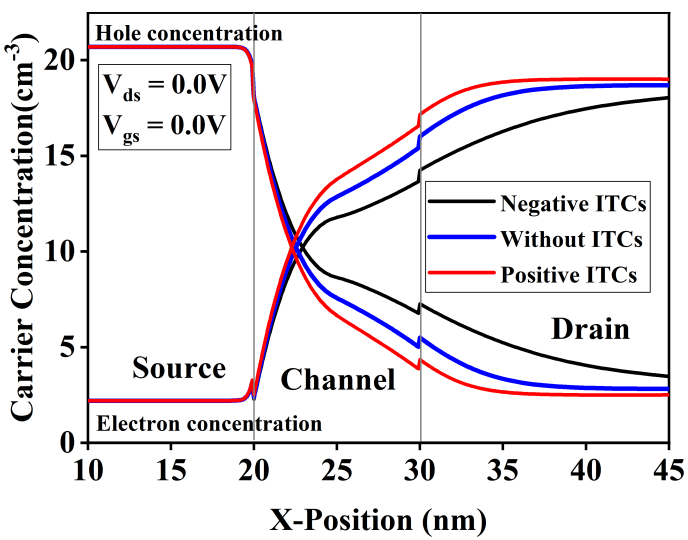

(a)

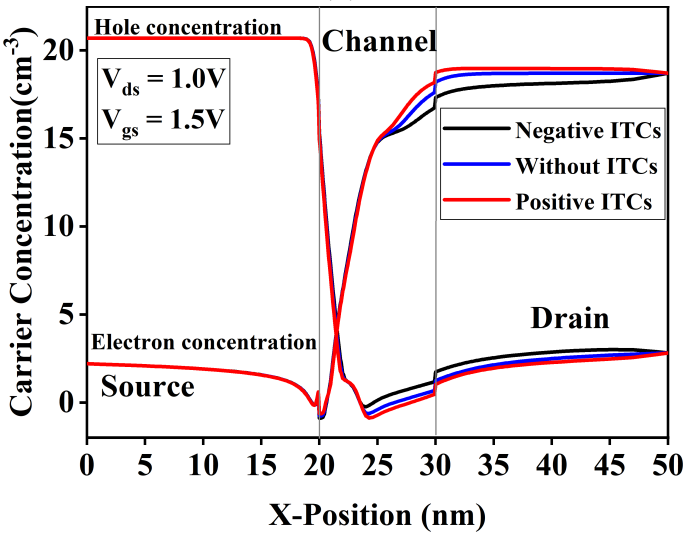

(c)

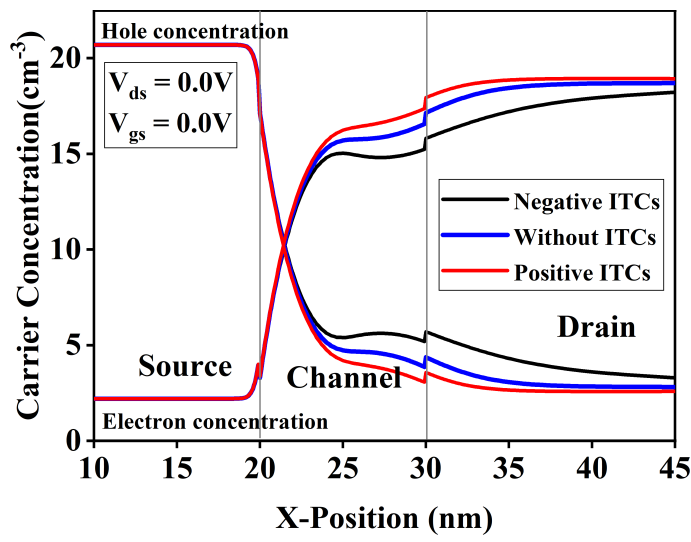

(b)

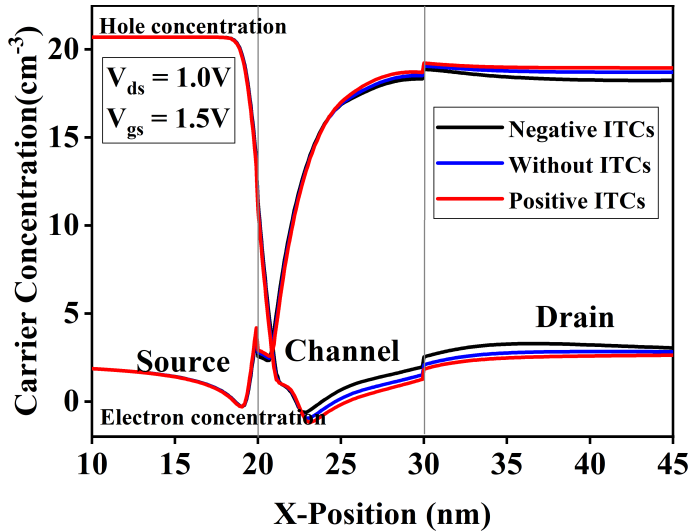

(d)

Fig. 3: Impact of ITC on (a) Carrier Concentration of DMG SC-TFET under Thermal Equilibrium (b) Carrier Concentration of HD DMG SC-TFET under Thermal Equilibrium (c) Carrier Concentration of DMG SC-TFET under ON-State (d) Carrier Concentration of HD DMG SC-TFET under ON-State

\section{Result and discussion}

In this section, an investigation is carried out to examine the impact of ITCs on physical characteristics, DC characteristics and analog/RF parameters for both simulated TFETs. Moreover, advance wireless communication system devices must behave linearly towards applied voltage. Hence, a comparative study of the linearity parameter is also carried out to determine device susceptibility toward reliable communication.

\subsection{Physical Characteristics}

This section discuss the physical characteristics of device illustrated in Fig 1. The study have been done under thermal equilibrium and ON-state with impact of positive ITCs, without ITCs and negative ITCs.

\subsubsection{Energy Band}

The impact of ITCs on energy band as function of device length are illustrated in Fig.2. Under thermal equilibrium, it is clearly observed from Fig. 2a,2b, tunneling width for charge carrier is wider that not allows to tunnel the majority carrier through junction. Also due to positive ITCs there is accumulation of electron above the valence band that allows downward shift of band, where as due to negative ITCs there accumulation of electron below the conduction band that allows upward shift of energy bands. Under ON-state, when we applied proper biasing to device, tunneling barrier for the charge carrier gets reduces as shown in Fig. 2c,2d. Moreover, it noticed that tunneling barrier of proposed TFET is lower then DMG SC-TFET because of the heterogeneous dielectric at the oxide layer which enhance the tunneling operation of the dominate charge carrier at the junction. 


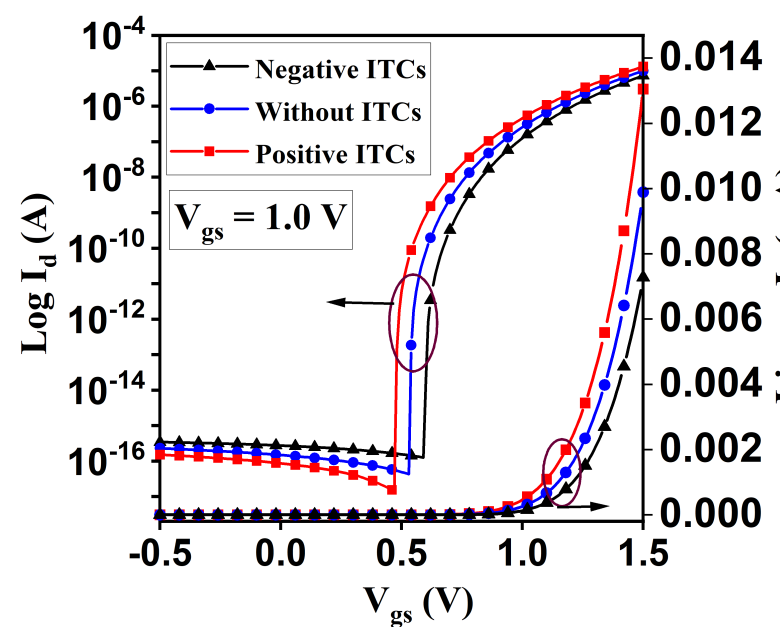

(a)

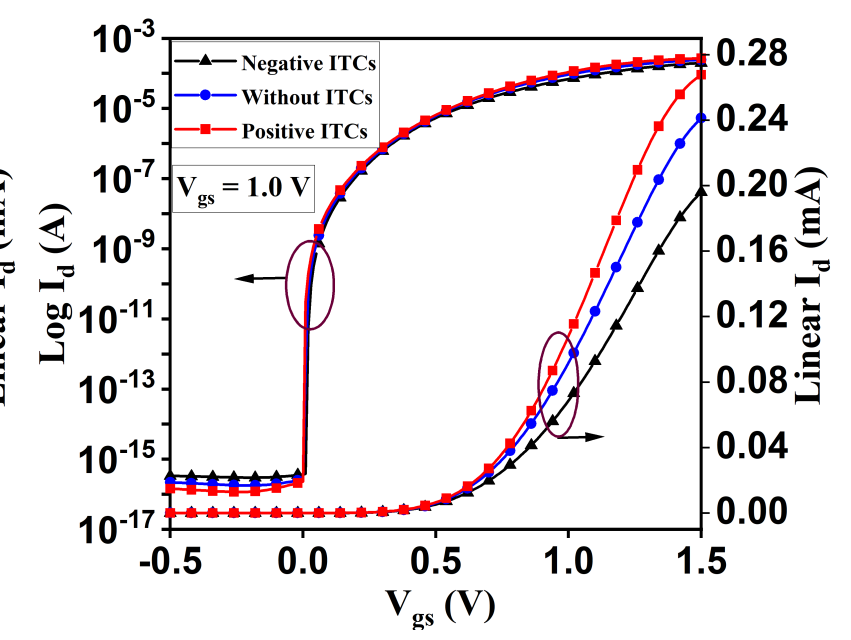

(b)

Fig. 4: Impact of ITCs on transfer characteristics (a) DMG SC-TEFT (b) HD DMG SC-TEFT

\subsubsection{Carrier Concentration}

Under thermal equilibrium, Fig. 3a ,3b shows the carrier (i.e, electron and hole) concentration along with channel direction where it is observed that symmetric carrier concentration in channel region with no accumulation of electron concentration in channel. Due to impact positive (negative) ITCs carrier (i.e, electron and hole) concentration get increases (decreases) in channel region as discuss earlier. Also it is notices that HD DMG SC-TFET exhibits more no number of carrier concentration in channel region as compared to DMG SC-TFET which is due to present of high- $\kappa$ dielectric material at the SC-junction oxide layer. Under ON-state condition, carrier concentration in channel region is observed from Fig $3 \mathrm{c}, 3 \mathrm{~d}$. This is due to narrowing of tunneling barrier width across the junctions that enhance the flow of charge carrier and hence charge concentration gets accumulates that leads to output current.

\subsection{Characteristics}

\subsubsection{Transfer Characteristics}

It represent the relationship between input voltage (i.e, $\mathrm{V}_{\mathrm{gs}}$ ) and output current (i.e, $\mathrm{I}_{\mathrm{d}}$ ). Fig.4 illustrates the impact of the ITCs on transfer characteristics for both TFETs on logarithmic and linear scale. Due to positive (negative) ITCs current driving capability (i.e, $\mathrm{I}_{\mathrm{d}}$ ) of both TFET devices increases (decreases). Because it improves (reduces) the energy band bending of the conduction band and valence band which results in decreased (increased) tunneling barrier. So, the proba- bility of tunneling charge carriers gets improved (reduced) which results in a increase (decrease) in currentcarrying capability. It is further observed that HD DMG SC-TFET achieves higher value $I_{d}$. This is due to the presence of $\mathrm{HfO}_{2}$ oxide layer at SC-junction which provides better gate coupling that leads to increased tunneling probability of majority charge carrier at the junctions. Moreover, the transfer characteristics of HD DMG SC-TFET are lesser influence by trap charges as compared to DMG SC-TFET because of high tunneling rate of charge carriers.

\subsubsection{Threshold Voltage $\left(V_{t h}\right)$}

For low power, high-frequency application, $\mathrm{V}_{\text {th }}$ of the device must be as low as possible. It is the minimum amount of voltage that is required to start tunneling phenomena at the junction that results in the current carrying capability of the device. Table 2 shows the comparison of $\mathrm{V}_{\mathrm{th}}$ for both device and it is notice the proposed device shows lower $\mathrm{V}_{\text {th }}$ due to high- $\kappa$ dielectric at the SC-junction which provides superior gate coupling at the junction with enhanced tunneling phenomena of the charge carrier that results in low $V_{\text {th }}$. Moreover, due to positive (negative) ITCs $\mathrm{V}_{\mathrm{th}}$ of the devices decreases (increases) due to degradation (enhancement) of tunneling width at the junction.

\subsubsection{Subthreshold Swing}

To achieves high switching speed, the device must have steeper subthreshold swing that results in high fanouts with reduced power consumption. The impact of ITCs on the subthreshold swing is depicted in Table 2 
Table 2: Impact of ITCs on Threshold Voltage, Subthreshold Swing and $\mathrm{I}_{\mathrm{on}} / \mathrm{I}_{\mathrm{off}}$ Ratio

\begin{tabular}{|c|c|c|c|c|}
\hline Parameters & Device & +ve ITCs & without ITCs & -ve ITCs \\
\hline \multirow{2}{*}{ Threshold Voltage } & DMG SC-TFET & $0.78 \mathrm{~V}$ & $0.80 \mathrm{~V}$ & $0.81 \mathrm{~V}$ \\
\cline { 2 - 5 } & HD DMG SC-TFET & $0.23 \mathrm{~V}$ & $0.24 \mathrm{~V}$ & $0.28 \mathrm{~V}$ \\
\hline \multirow{2}{*}{ Subthreshold Swing } & DMG SC-TFET & $2.51 \mathrm{mV} /$ decade & $2.75 \mathrm{mV} /$ decade & $4.58 \mathrm{mV} /$ decade \\
\cline { 2 - 5 } & HD DMG SC-TFET & $1.95 \mathrm{mV} /$ decade & $3.09 \mathrm{mV} /$ decade & $2.38 \mathrm{mV} /$ decade \\
\hline \multirow{2}{*}{$\mathrm{I}_{\text {on }} / \mathrm{I}_{\text {off }}$ Ratio } & DMG SC-TFET & $1.52 \times 10^{11}$ & $6.68 \times 10^{10}$ & $2.65 \times 10^{10}$ \\
\cline { 2 - 5 } & HD DMG SC-TFET & $1.16 \times 10^{12}$ & $9.05 \times 10^{11}$ & $5.28 \times 10^{11}$ \\
\hline
\end{tabular}

and it notices proposed devices have high subthreshold swing as compared to DMG SC-TFET. Moreover, due to positive (negative) ITCs it's values get decreases (increases) for HD DMG SC-TFET. Thus, the positive (negative) trap charge improves (degrades) the device performance.

\subsection{4 $I_{\text {on }} / I_{\text {off }}$ Ratio}

In order to achieves high switching speed, device must have high $\mathrm{I}_{\mathrm{on}} / \mathrm{I}_{\text {off }}$ ratio. For this, device must have high ON-state current $\left(\mathrm{I}_{\mathrm{on}}\right)$ and low OFF-state current $\left(\mathrm{I}_{\mathrm{off}}\right)$. Table 2 manifest the impact of ITCs on $\mathrm{I}_{\mathrm{on}} / \mathrm{I}_{\text {off }}$ ratio and it is observe due to positive (negative) ITC $\mathrm{I}_{\mathrm{on}} / \mathrm{I}_{\mathrm{off}}$ ratio result in increases (decreases) in value. Moreover, proposed device shows higher $\mathrm{I}_{\mathrm{on}} / \mathrm{I}_{\text {off }}$ ratio and lesser impact of ITCs in comparison to DMG SC-TFET. This makes appropriate device for ultra-low power high frequency application.

\subsection{Analog/RF Parameters}

In this section, a detailed comparative studies are carried out to investigates the impact of trap charges on analog/RF parameters of both the simulated TFET. These parameters play a most important role in determining device performance at high-frequency. For better reliability and good life spam, these parameters should have less impact on ITCs.

\subsubsection{Gate-to-Drain capacitance $\left(C_{g d}\right)$}

$\mathrm{C}_{\mathrm{gd}}$ is dominating parasitic capacitance that determines analog/RF performance parameters like cut-off frequency. Expression for $\mathrm{C}_{\mathrm{gd}}$ is given by equation 1 [26]. Fig. 5 manifests a graphical representation of $\mathrm{C}_{\mathrm{gd}}$ with respect to $\mathrm{V}_{\mathrm{gs}}$ at different traps charges. $\mathrm{C}_{\mathrm{gd}}$ of devices increase with $\mathrm{V}_{\mathrm{gs}}$ due to decrease drain-channel barrier. Moreover, due to positive (negative) traps charges $\mathrm{C}_{\mathrm{gd}}$ increases (decreases) because it increases (decreases) inversion layer in the channel region from drain to source. From these results, it observed that proposed TFET shows lesser influences of trap charges due to heterogeneous dielectric material at the oxide layer which provides proper gate coupling at the junctions.

$C_{\mathrm{gd}}=\frac{\partial Q_{\mathrm{g}}}{\partial V_{\mathrm{gd}}}$

\subsubsection{Transconductance $\left(g_{m}\right)$}

$\mathrm{g}_{\mathrm{m}}$ is an essential parameter for examining device performance which is express by equation 2 [27]. $\mathrm{g}_{\mathrm{m}}$ shows the electrical characteristics of the device that relates the $I_{d}$ to applied $V_{g s}$ and determine the current driving capability of the device. For better device performance we required high value of $g_{m}$. Fig 6 illustrate the impact of ITCs on $g_{m}$ with respect to $V_{g s}$. Since $g_{m}$ is directly proportional to $I_{d}$, therefore it increases with applied $\mathrm{V}_{\mathrm{gs}}$ for both the devices but for the HD DMG SC-TFET it starts to decrease after achieves maximum peak due current saturation. Moreover, due to impact of positive (negative) ITCs plots of $g_{m}$ shift upward (downward) because value of $I_{d}$ increases (decreases) that leads to upgradation (degradation) analog/RF performance parameters.

$g_{\mathrm{m}}=\frac{\partial I_{\mathrm{d}}}{\partial V_{\mathrm{gs}}}$

\subsubsection{Cut-off Frequency $\left(f_{T}\right)$}

$\mathrm{f}_{\mathrm{T}}$ is the most dominant performance parameter for high-frequency low power devices. According to equation $3[28] \mathrm{f}_{\mathrm{T}}$ of device is directly dependent to $\mathrm{g}_{\mathrm{m}}$ and inversely dependent to total parasitic capacitance (i.e, $\mathrm{C}_{\mathrm{gd}}, \mathrm{C}_{\mathrm{gs}}$ ) where $\mathrm{g}_{\mathrm{m}}$ is dominating factor. Fig. 7 depicts proposed device have higher value as compared to DMG SC-TFET which is due to $\mathrm{HfO}_{2}$ present at SC-junction which enhance tunneling phenomena. As $\mathrm{V}_{\mathrm{gs}}$ increases, $\mathrm{f}_{\mathrm{T}}$ of the both devices increases due to increment in $\mathrm{g}_{\mathrm{m}}$ and for proposed device after attain maximum peak it start to decrease due increase in $\mathrm{C}_{\mathrm{gd}}$ and mobility saturation of charge carrier. Moreover, we have observed impact of ITCs and notice $\mathrm{f}_{\mathrm{T}}$ of the devices increases 


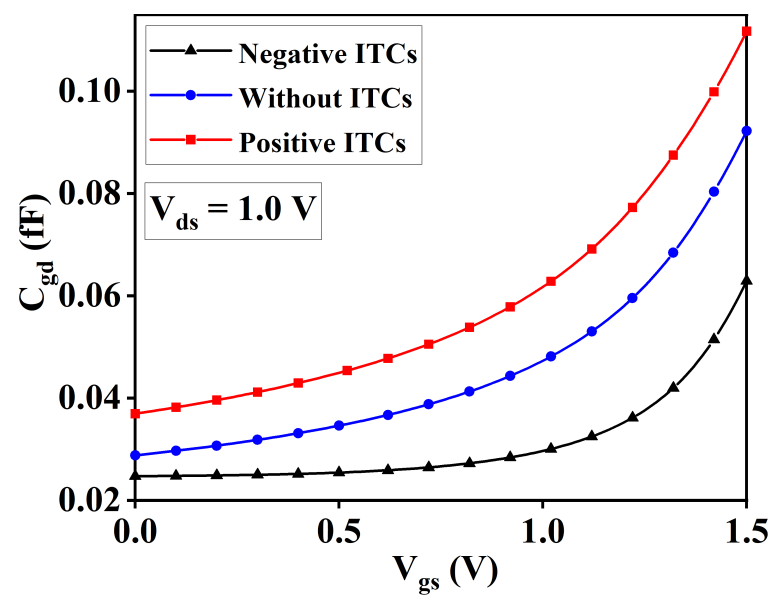

(a)

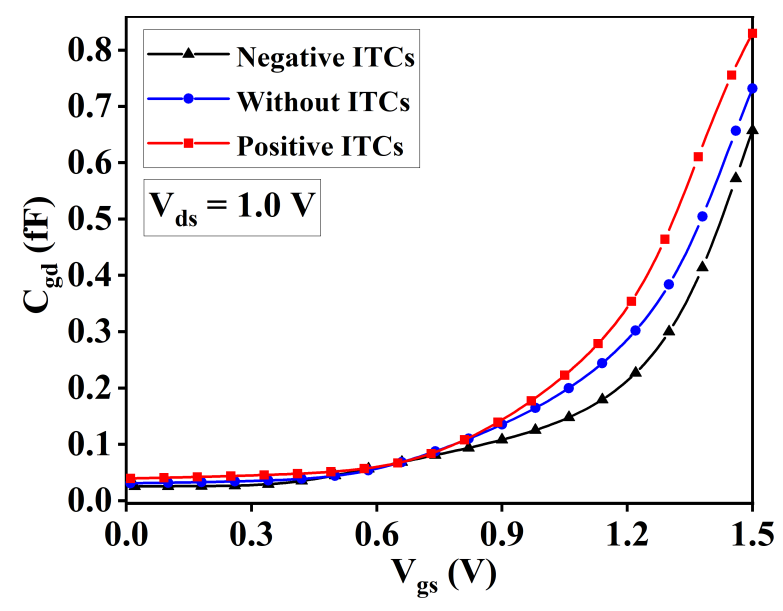

(b)

Fig. 5: Impact of ITCs on $\mathrm{C}_{g d}$ (a) DMG SC-TEFT (b) HD DMG SC-TEFT

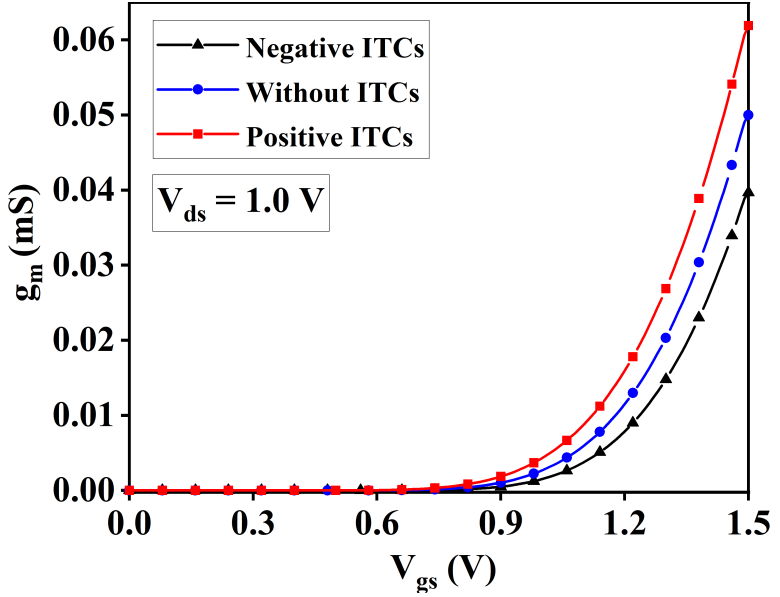

(a)

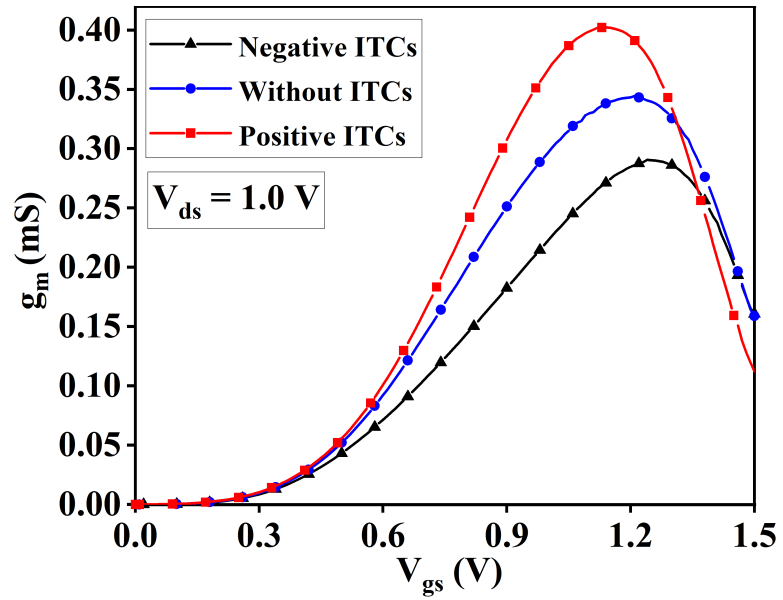

(b)

Fig. 6: Impact of ITCs on $g_{m}$ (a) DMG SC-TEFT (b) HD DMG SC-TEFT

(decreases) with positive (negative) ITCs similar to $\mathrm{g}_{\mathrm{m}}$ curve.

$f_{\mathrm{T}}=\frac{g_{\mathrm{m}}}{2 \pi\left(C_{\mathrm{gd}}+C_{\mathrm{gs}}\right)}$

\subsubsection{Gain Bandwidth Product (GBP)}

GBP is also most essential performance parameter to examine the device performance. Expression of GBP is given by equation 4 [28] which shows GBP is directly dependent to $\mathrm{g}_{\mathrm{m}}$ and inversely dependent to $\mathrm{C}_{\mathrm{gd}}$. The impact of ITCs on GBP illustrates in Fig. 8 where proposed device exhibit the higher value of GBP and it increases with $\mathrm{V}_{\mathrm{gs}}$ due to high- $\kappa$ dielectric material at SC-junction that reduces the tunneling barrier and enhance the tunneling phenomena of charge carrier which results in higher $g_{m}$ and after attaining maximum peak it starts to decreases due to scattering mechanism of charge carrier at high $\mathrm{V}_{\mathrm{gs}}$. Moreover, due to the positive (negative) ITCs it achieves higher (lower) peak similarly to $g_{m}$ due to a direct relationship.

$G B P=\frac{g_{\mathrm{m}}}{2 \pi C_{\mathrm{gd}}}$

\subsubsection{Transconductance Generation Factor (TGF)}

TGF is the performance parameter that determines device efficiency to convert $I_{d}$ into $g_{m}$ which measures the ability to convert power into speed. For better performance and low power consumption we required a higher value of TGF. The expression for TGF is given by equation 5 [29]. Fig. 9 manifest comparatives plot for the 


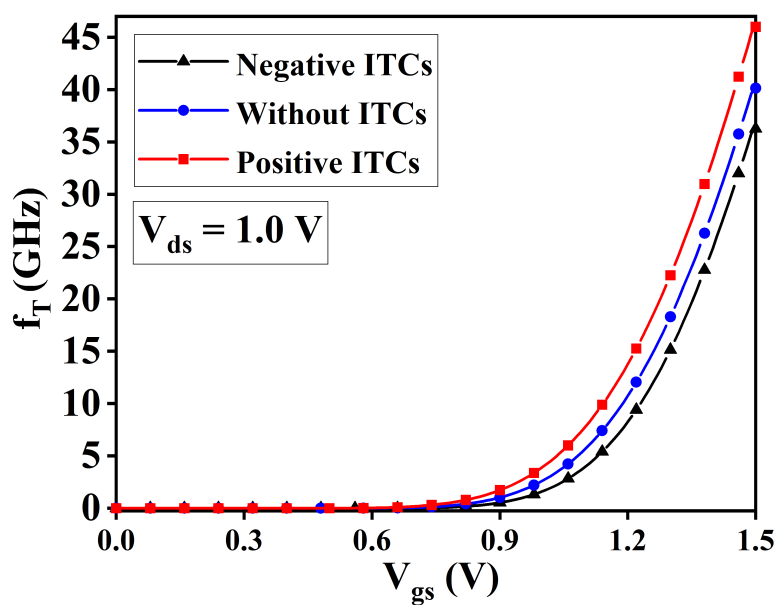

(a)

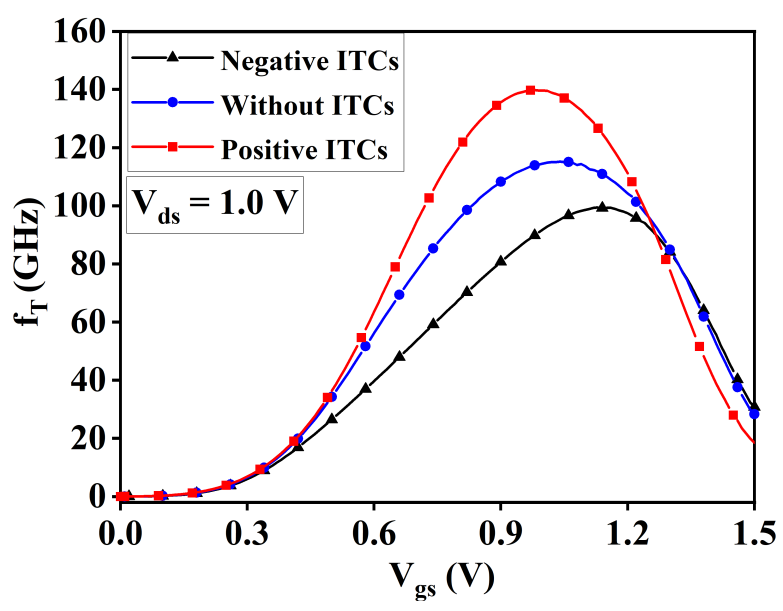

(b)

Fig. 7: Impact of ITCs on $\mathrm{f}_{\mathrm{T}}$ (a) DMG SC-TEFT (b) HD DMG SC-TEFT

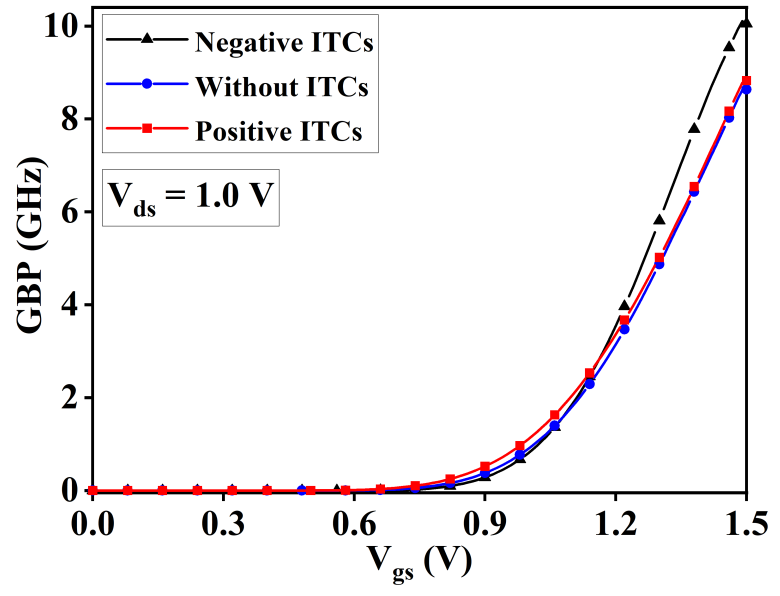

(a)

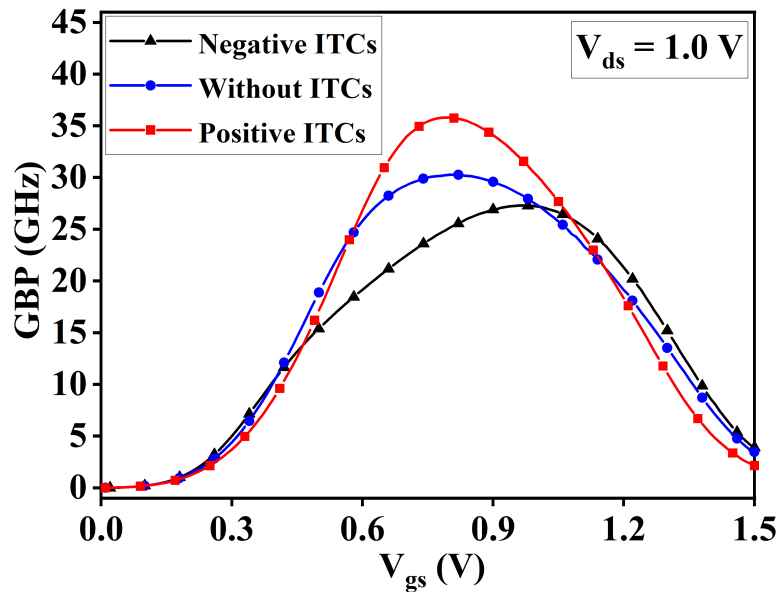

(b)

Fig. 8: Impact of ITCs on GBP (a) DMG SC-TEFT (b) HD DMG SC-TEFT

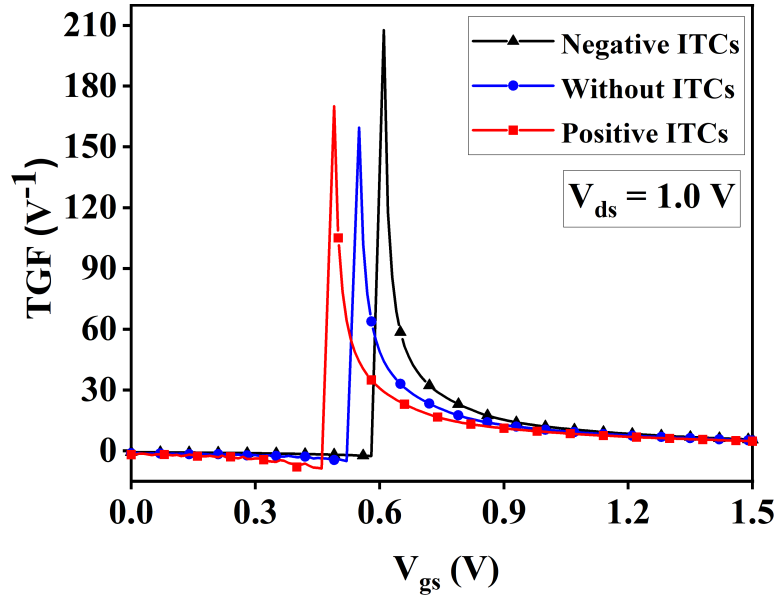

(a)

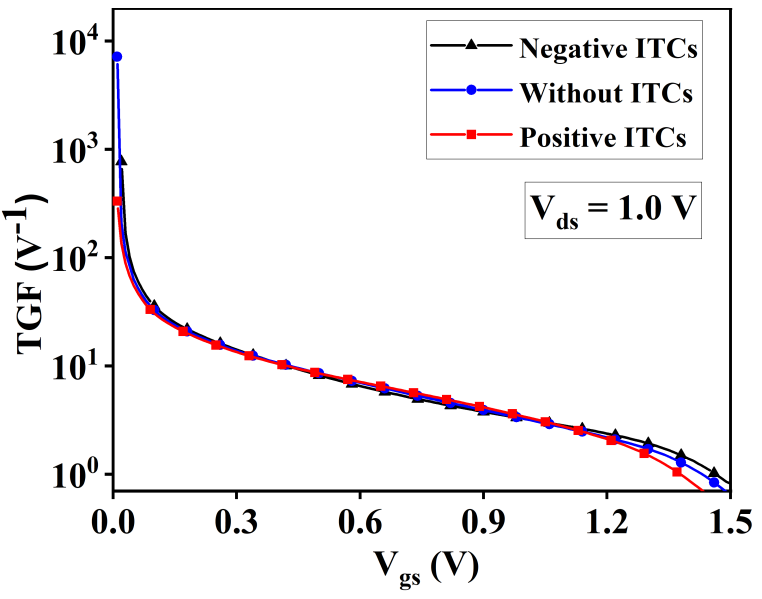

(b)

Fig. 9: Impact of ITCs on TGF (a) DMG SC-TEFT (b) HD DMG SC-TEFT 


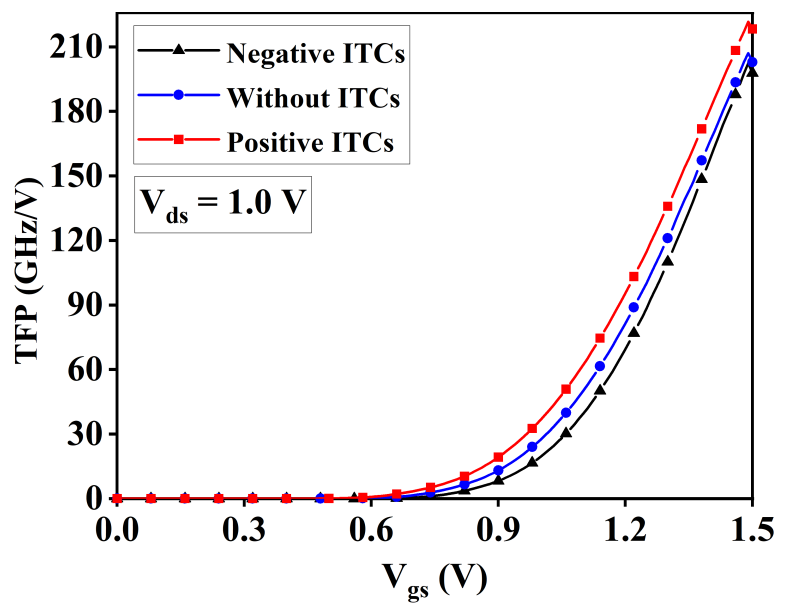

(a)

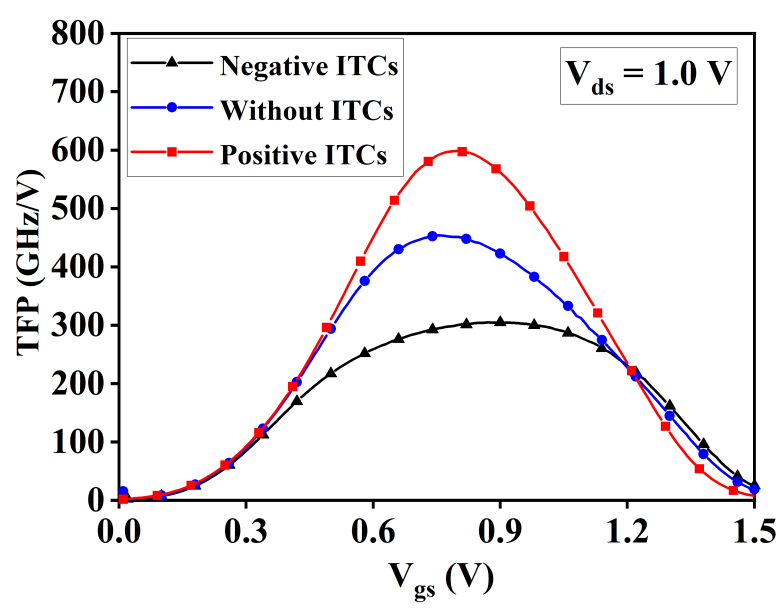

(b)

Fig. 10: Impact of ITCs on TFP (a) DMG SC-TEFT (b) HD DMG SC-TEFT

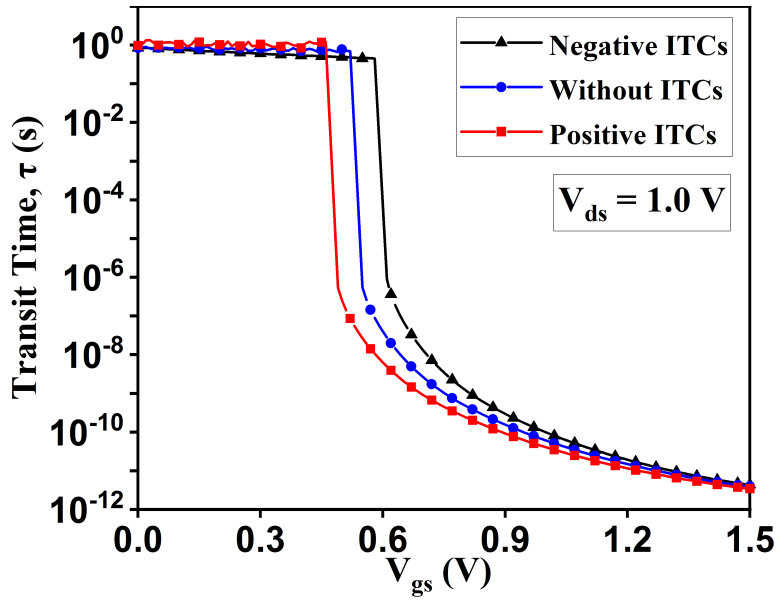

(a)

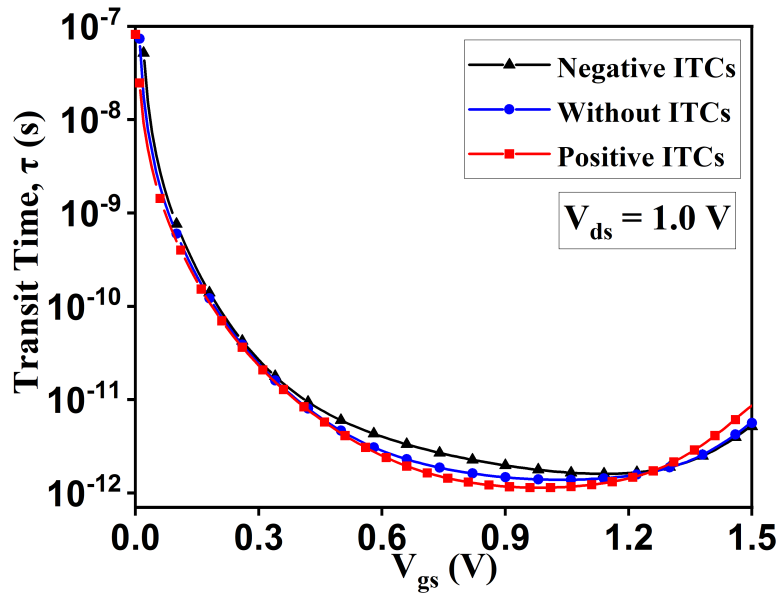

(b)

Fig. 11: Impact of ITCs on $\tau$ (a) DMG SC-TEFT (b) HD DMG SC-TEFT

impact of ITCs on TGF with a change in $\mathrm{V}_{\mathrm{gs}}$. It is observed proposed devices show higher TGF and almost negligible impact of ITCs due to the presence of $\mathrm{HfO}_{2}$ oxide layer at SC-junction which reduces the barrier width for charge carrier and result in increase tunneling probability at the junction. In general, TGF gets decreases with increases in $\mathrm{V}_{\mathrm{gs}}$ but DMG SC-TFET shows significant behavior for the lower value of $V_{g s}$ due to high value of $\mathrm{V}_{\mathrm{th}}$.

$T G F=\frac{g_{\mathrm{m}}}{I_{\mathrm{d}}}$

\subsubsection{Transconductance Frequency Product (TFP)}

TFP is another most crucial parameter that determines device performance at the operating frequency. It shows the relationship between power and bandwidth for highspeed device applications. For better device performance it must be high as possible. Expression for TFP is given by equation 6 which shows direct relationship with $\mathrm{f}_{\mathrm{T}}$ [29]. The impact of ITCs on TFP with applied $\mathrm{V}_{\mathrm{gs}}$ is illustrated in Fig. 10. It is clearly noticed that TFP of the proposed device is higher than DMG SC-TFET and it increases with increases value of $\mathrm{V}_{\mathrm{gs}}$. And once it attain maximum peak it starts to decreases due to degraded mobility of charge carrier at high $\mathrm{V}_{\mathrm{gs}}$. Moreover, due to impact positive (negative) ITCs it attains a higher (lower) peak similar to $\mathrm{f}_{\mathrm{T}}$.

$T F P=\frac{g_{\mathrm{m}} f_{\mathrm{T}}}{I_{\mathrm{d}}}$

\subsubsection{Transit Time $(\tau)$}

It is the most important parameter for examining the device speed to evaluate circuit performance. For high- 


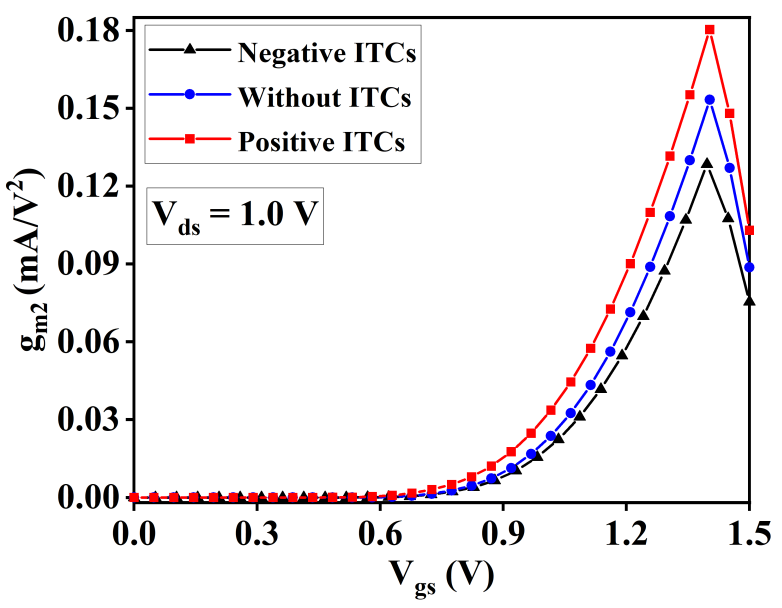

(a)

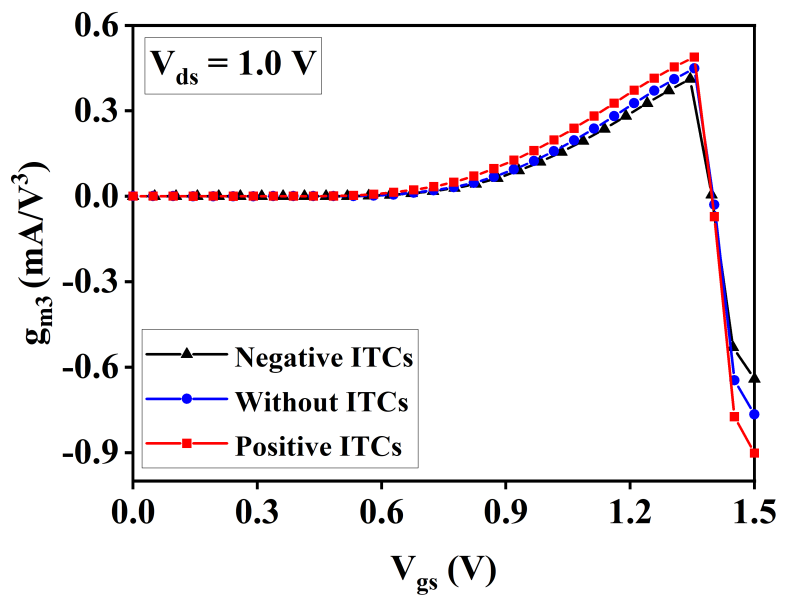

(c)

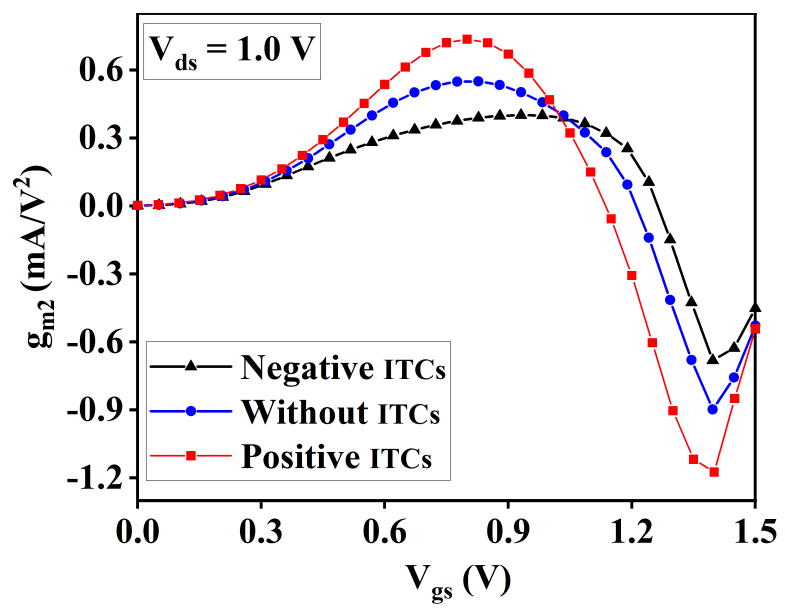

(b)

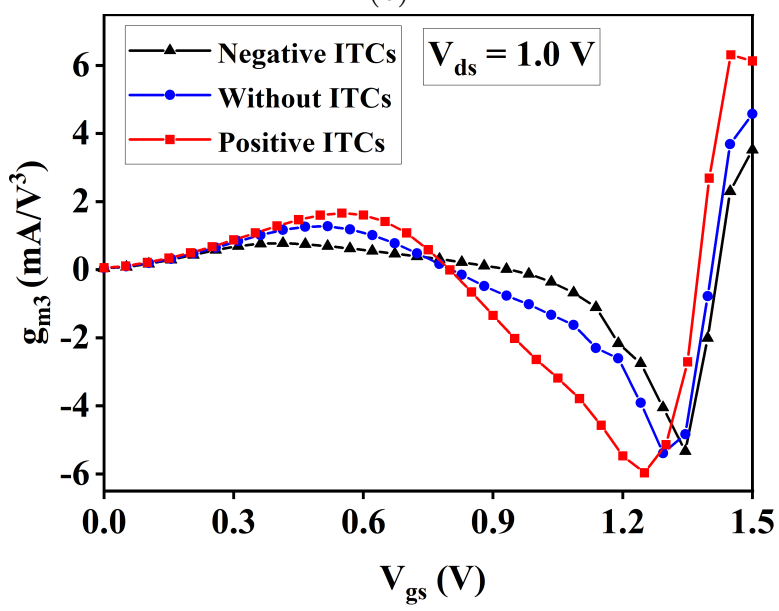

(d)

Fig. 12: Impact of ITCs on (a) $g_{m 2}$ of DMG SC-TEFT (b) $g_{m 2}$ of HD DMG SC-TEFT (c) $g_{m}$ of DMG SC-TEFT (d) $g_{\mathrm{m} 3}$ of HD DMG SC-TEFT

speed performance we require less $\tau$ for less delay in performance speed. Expression for $\tau$ is given by equation 7 which shows inverse relationship with $\mathrm{f}_{\mathrm{t}}$ [30]. The impact of ITCs on $\tau$ is illustrated in Fig. 11 where it noticed the proposed device shows lesser $\tau$ as compared to DMG SC-TFET due to higher value of $\mathrm{f}_{\mathrm{t}}$. Moreover, due to positive (negative) ITCs it gets decreases (increases) and HD DMG SC-TFET shows the negligible effect of ITCs due to presence of $\mathrm{HfO}_{2}$ oxide layer at SC-junction which increases the flow of charge carrier due to proper gate coupling at the junction. This makes a very promising TFET for low power high-frequency devices.

$\tau=\frac{1}{2 \pi f_{\mathrm{T}}}$

\subsection{Linearity Analysis}

In this scenario, along with high-speed wireless communication systems needs a device that has less noise and high signal to noise ratio to attains maximum linearity performance with reduces power consumption. Higher linearity performance means low distortion at the output with applied $\mathrm{V}_{\mathrm{gs}}$. If the linearity performance is not maintained by the system then non-linearity behavior induced noise/distortion at the output. And it is seen in many studies that ITCs widely influence the linearity performance of the TFET. So, it is important to assessment the impact of ITCs on the linearity parameters of the devices.

\subsubsection{Higher Order Transconcuctance}

Non-linearity behavior of device examine from higher order transconcuctance that are $2^{\text {nd }}$ order and $3^{\text {rd }}$ or- 


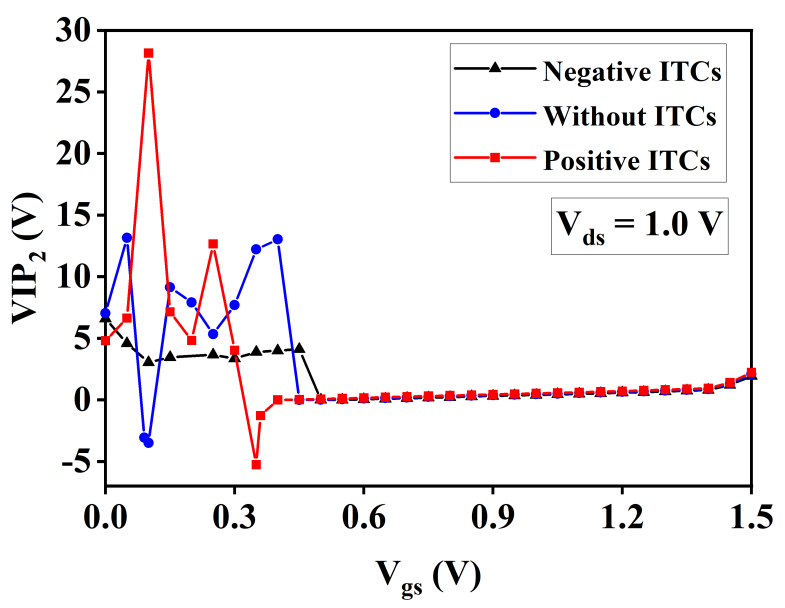

(a)

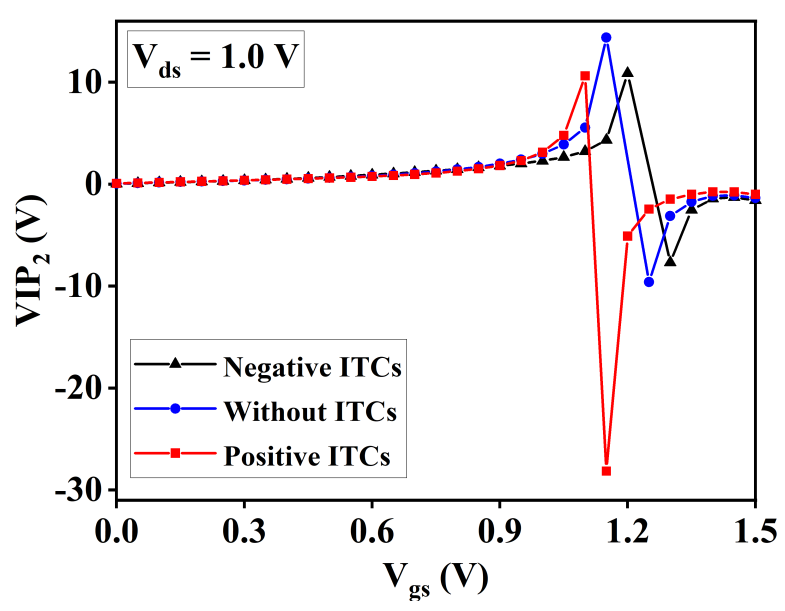

(b)

Fig. 13: Impact of ITCs on $\operatorname{VIP}_{2}$ (a) DMG SC-TEFT (b) HD DMG SC-TEFT

der derivative of $I_{d}$ with respect to $V_{g s}$ i.e, $g_{m 2}$ and $\mathrm{g}_{\mathrm{m} 3}$ which are given by equation 8 and 9 respectively [31]. For better linearity performance they must have low peaks with applied $\mathrm{V}_{\text {gs }}$. The impact of ITCs on higher-order transconductance is illustrated in Fig 12 and it observed the proposed device shows lower peaks. Due to positive ITCs zero crossover frequency moves toward higher peaks that implies higher $\mathrm{V}_{\mathrm{gs}}$ is required to maintain linear behavior of the device. The degradation of the linearity parameter for positive ITCs is due to enhancement in SCE characteristics of device which increases with positive ITCs. Moreover, due to negative ITCs zero crossover frequency shifts downward that increase the device performance.

$g_{\mathrm{m} 2}=\frac{\partial^{2} I_{\mathrm{d}}}{\partial^{2} V_{\mathrm{gs}}}$

$g_{\mathrm{m} 3}=\frac{\partial^{3} I_{\mathrm{d}}}{\partial^{3} V_{\mathrm{gs}}}$

\subsubsection{Second Order Voltage Intercept Point (VIP 2$)$}

$\mathrm{VIP}_{2}$ examine mathematically input voltage (i.e. $\mathrm{V}_{\mathrm{gs}}$ ) at which first and second order harmonic voltages become equal. Expression for $\mathrm{VIP}_{2}$ is given by equation 10 [32]. For better performance peaks of $\mathrm{VIP}_{2}$ must be high. Fig. 13 manifests the impact of ITCs on $\mathrm{VIP}_{2}$ and it noticed the proposed device shows high peaks in comparison to DMG SC-TFET. This is due to $\mathrm{HfO}_{2}$ oxide layer at the SC-junction that's allow higher tunneling phenomena at the junction. However due to positive (negative) trap charges $\mathrm{VIP}_{2}$ of HD DMG SC-TFET achieves a higher peak earlier (later) as compared to without ITCs. And DMG SC-TFET shows some random peaks at low $\mathrm{V}_{\mathrm{gs}}$ due to high $\mathrm{V}_{\mathrm{th}}$.

$V I P_{2}=4\left(\frac{g_{\mathrm{m}}}{g_{\mathrm{m} 2}}\right)$

\subsubsection{Third Order Voltage Intercept Point $\left(\mathrm{VIP}_{3}\right)$}

$\mathrm{VIP}_{3}$ examine mathematically input voltage (i.e. $\mathrm{V}_{\mathrm{gs}}$ ) at which first and third order harmonic voltages become equal. Similarly to $\mathrm{VIP}_{2}$ peaks of $\mathrm{VIP}_{3}$ needs to high for distortion-less output. Expression for $\mathrm{VIP}_{3}$ is given by equation 11 [32]. The impact of ITCs on $\mathrm{VIP}_{3}$ is illustrated in Fig. 14 and it is noticed the proposed device have high peaks in comparison to DMG SC-TFET. And DMG SC-TFET shows random peaks at low applied $\mathrm{V}_{\mathrm{gs}}$. Moreover, due to positive ITCs proposed device shows the maximum peak which is clearly shown in plots. Also, due to negative ITCs it shows a higher peak as compared to without interface trap charges. Hence, due to the impact of trap charges device performance gets improved.

$V I P_{3}=\sqrt{24\left(\frac{g_{\mathrm{m}}}{g_{\mathrm{m} 3}}\right)}$

\subsubsection{Third Order Input Intercept Point (IIP 3$)$}

$\mathrm{IIP}_{3}$ examine mathematically input power at which first and second order harmonic powers become equal. Expression for $\mathrm{IIP}_{3}$ is given by equation 12 [32]. For reliable communication IIP $_{3}$ peaks should be high as possible. Fig. 15 illustrated the ITCs impact on $\operatorname{IIP}_{3}$ with applied $\mathrm{V}_{\mathrm{gs}}$ and it observed the proposed device shows higher peaks at low applied $\mathrm{V}_{\mathrm{gs}}$ in comparison to DMG 


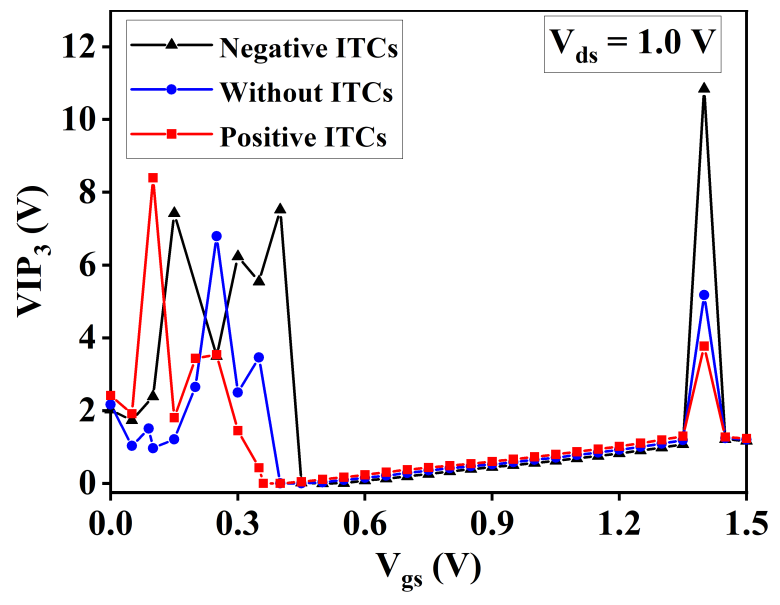

(a)

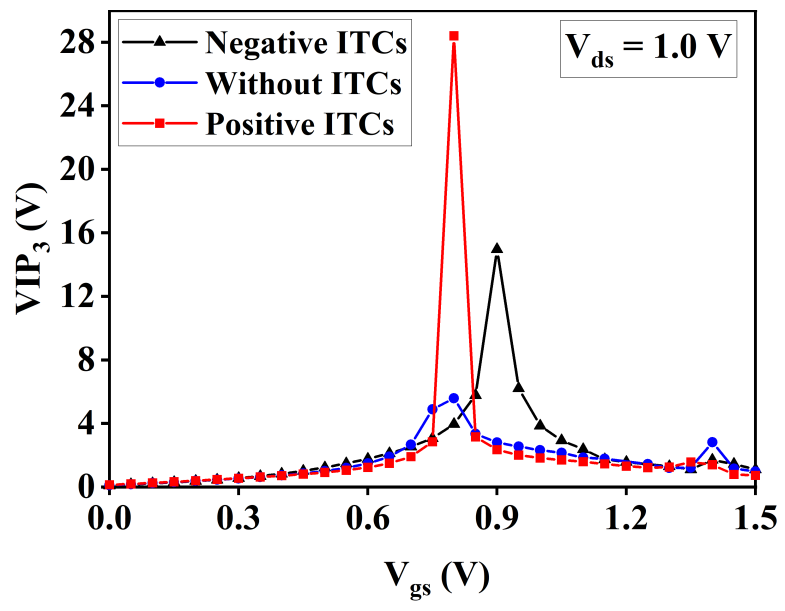

(b)

Fig. 14: Impact of ITCs on $\mathrm{VIP}_{3}$ (a) DMG SC-TEFT (b) HD DMG SC-TEFT

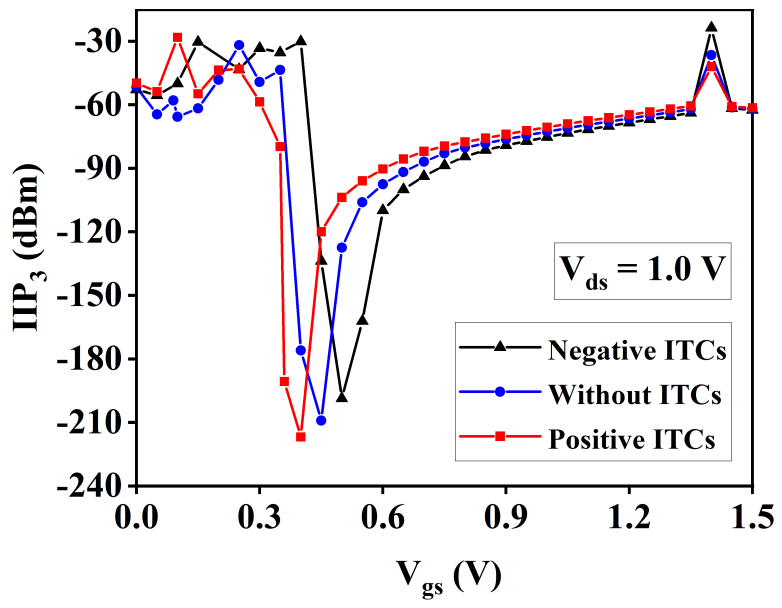

(a)

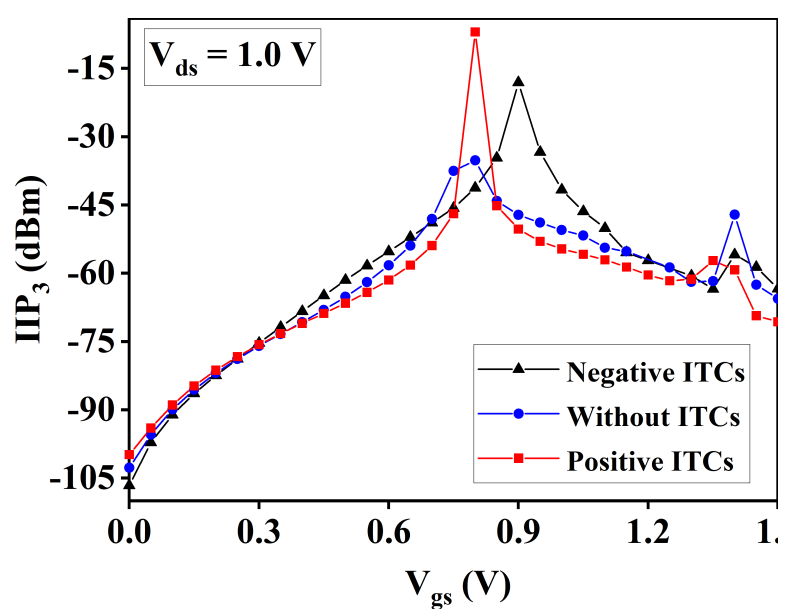

(b)

Fig. 15: Impact of ITCs on $\mathrm{IIP}_{3}$ (a) DMG SC-TEFT (b) HD DMG SC-TEFT

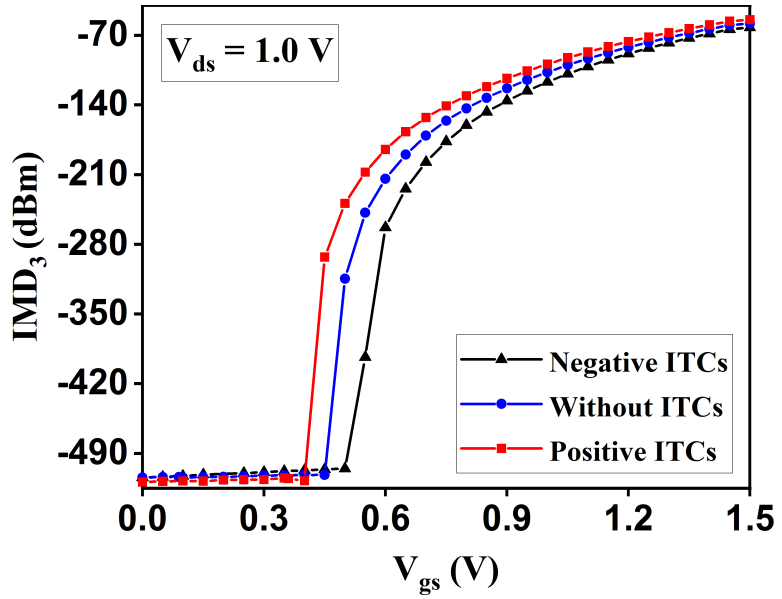

(a)

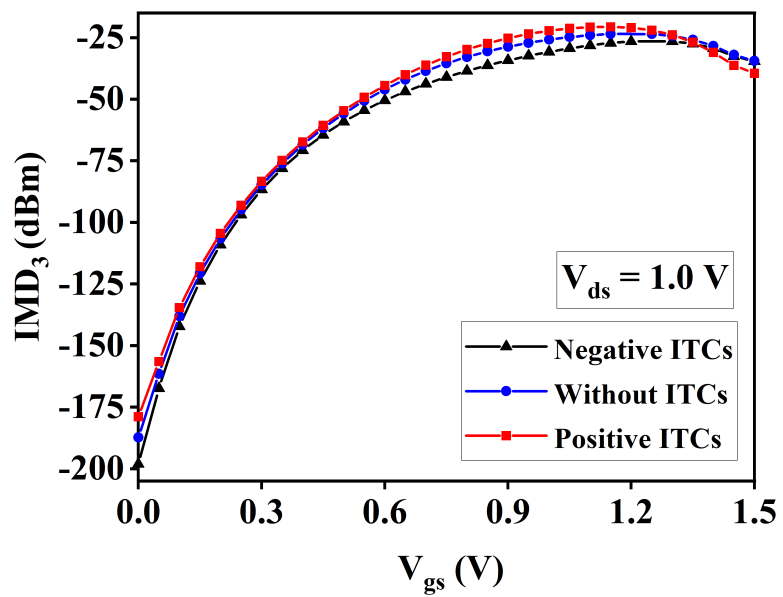

(b)

Fig. 16: Impact of ITCs on $\mathrm{IMD}_{3}$ (a) DMG SC-TEFT (b) HD DMG SC-TEFT 


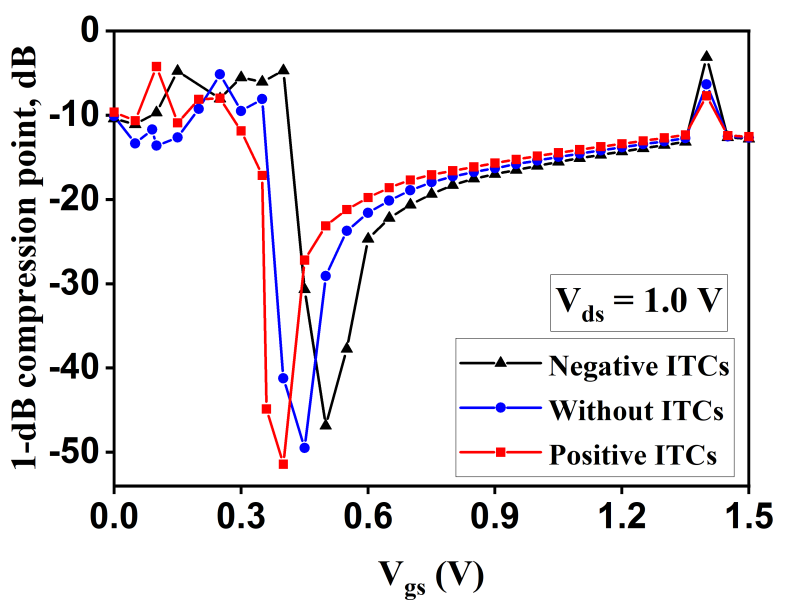

(a)

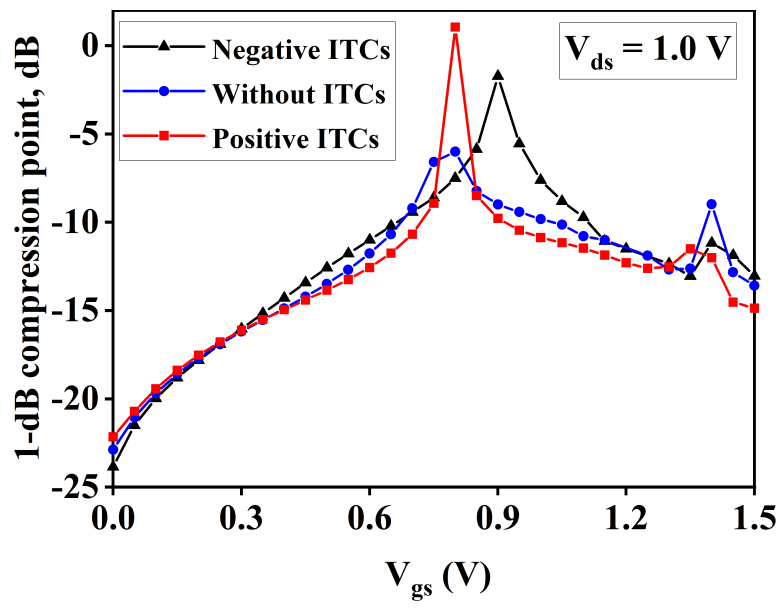

(b)

Fig. 17: Impact of ITCs on 1dB compression point (a) DMG SC-TEFT (b) HD DMG SC-TEFT

SC-TFET due to $\mathrm{HfO}_{2}$ oxide layer at SC-junction which increases tunneling of charge carrier. It is further notices, due to the impact of ITCs peaks of $\operatorname{IIP}_{3}$ increases because of increased tunneling phenomena at the junction and DMG SC-TFET shows some random peaks at low $\mathrm{V}_{\mathrm{gs}}$ due to high $\mathrm{V}_{\mathrm{th}}$.

$I I P_{3}=\frac{2}{3}\left(\frac{g_{\mathrm{m}}}{g_{\mathrm{m} 3} R_{\mathrm{s}}}\right)$

where, $\mathrm{R}_{\mathrm{s}}=50 \Omega$ for $\mathrm{RF} /$ analog application

\subsubsection{Third Order Intermodulation Distortion (IMD 3 )}

$\mathrm{IMD}_{3}$ generally determines intermodulation distortion which occurs due to the non-linearity response of the device. Expression for $\mathrm{IMD}_{3}$ is given by equation 13 [32]. For distortion-less communication $\mathrm{IMD}_{3}$ values should be low with applied $V_{\text {gs }}$. The impact of ITCs on $\mathrm{IMD}_{3}$ is portrayed in Fig. 16 where it is noticed that proposed TFET shows higher value as compared to DMG SC-TFET. Also, due to positive (negative) ITCs it increases (decreases) and the proposed device shows the almost negligible impact of ITCs. Moreover it also observed, at low $\mathrm{V}_{\mathrm{gs}}$ the value of $\mathrm{IIP}_{3}$ is higher as compared to $\mathrm{IMD}_{3}$ which ensures that proposed device has superior linearity behavior with low distortion at applied $\mathrm{V}_{\mathrm{gs}}$ that makes a very promising device for the reliable communication system.

$I M D_{3}=\left[\frac{9}{2}\left(V I P_{3}\right)^{2} g_{\mathrm{m} 3}\right]^{2} R_{\mathrm{s}}$

\subsubsection{1dB Compression Point}

It is another most significant parameter to determine the linearity performance of the device. It defines input power level at which causes output power level shifts linearly from $1 \mathrm{~dB}$ which is given by equation 14 [32]. It used to determine maximum input power after which the gain of the device degraded. Fig 17 manifests the impact of trap charges on $1 \mathrm{~dB}$ compression point, revealing higher peak of proposed device in comparison to DMG SC-TFET. Moreover, due to positive and negative ITCs there are increases in $1 \mathrm{~dB}$ compression point that result in upgrade linearity performance. And DMG SC-TFET shows some random peaks at low applied $\mathrm{V}_{\mathrm{gs}}$ due to high $\mathrm{V}_{\mathrm{th}}$ of the device.

$1 d B$ compression point $=0.22 \sqrt{\frac{g_{\mathrm{m}}}{g_{\mathrm{m} 3}}}$

\section{Conclusion}

This research work present a novel TFET to overcome the shortcomings of conventional TFET. The proposed device employed combines benefits of both heterogeneous dielectric material and gate engineering which result in low $\mathrm{V}_{\mathrm{th}}$, high $\mathrm{I}_{\text {on }}$ in the order $10^{-4}$ with 3.09 $m V /$ decade subthreshold swing. In addition to it $\mathrm{I}_{\mathrm{on}} / \mathrm{I}_{\mathrm{off}}$ ratio in the order of $10^{11}$. This makes a very promising device for digital circuit applications. Generally, trap charges are induced at the interface of the semiconductor/oxide layer during the fabrication process and these ITCs degrades the device performance. So, to assessment of the impact of positive and negative ITCs a comparative investigation is done between DMG SC-TFET and proposed device. This study shows that HD DMG SC-TFET has higher $\mathrm{C}_{\mathrm{gd}}$ which is due to $\mathrm{HfO}_{2}$ material at $\mathrm{SC}$-junction. But, at the same times it shows large increment in $g_{m}$ that enhance the current capability of the device which shows very significant improvement 
in all other analog/RF parameters such as $f_{\mathrm{T}}, \mathrm{GBP}$, TGF, TFP, $\mathrm{f}_{\max }$ and $\tau$. Due to the impact of positive (negative) ITCs proposed TFET shows enhance (degrade) device performance and also exhibit better immunity towards trap charges in comparison to DMG SC-TFET. Since the linearity behavior of the device plays a very crucial role for distortion-less and noisefree wireless communications. Therefore, impact of trap charges on linearity parameters is also examine and it observed proposed device has better response in comparison to DMG SC-TFET that makes very promising device for advance communication system. These results prove that our proposed TFET is suitable for low power high-frequency electronic devices.

\section{Declarations}

The manuscript follows all the ethical standards, including plagiarism.

- Funding statement : Not applicable.

- Conflict of interest : No conflicts of interest.

- Authors contributions :

- Sachin Kumar: Conceptualization, data curation, formal analysis, methodology, investigation, writing - original draft.

- Dharmendra Singh Yadav: Supervision, validation, visualization, writing - review \& editing

- Availability of Data and Material : The data and material concerned to the manuscript may be made available on request.

- Consent for Publication : Yes.

- Consent to Participate : Yes.

- Acknowledgements : The authors would like to thank Dr. Dip Prakash Samajdar from Department of Electronics and Communication Engineering, PDPM Indian Institute of Information Technology, Design \& Manufacturing, Jabalpur, Madhya Pradesh, India for providing valuable suggestions and support to carry out this research work.

\section{References}

1. S.O. Koswatta, M.S. Lundstrom, D.E. Nikonov, Performance comparison between pin tunneling transistors and conventional mosfets, IEEE Transactions on Electron Devices 56(3), 456 (2009)

2. M.H. Tsai, T.P. Ma, The impact of device scaling on the current fluctuations in mosfet's, IEEE Transactions on Electron Devices 41(11), 2061 (1994)

3. S.W. Sun, P.G. Tsui, Limitation of cmos supply-voltage scaling by mosfet threshold-voltage variation, IEEE Journal of solid-state circuits 30(8), 947 (1995)
4. W.Y. Choi, B.G. Park, J.D. Lee, T.J.K. Liu, Tunneling field-effect transistors (tfets) with subthreshold swing (ss) less than $60 \mathrm{mv} / \mathrm{dec}$, IEEE Electron Device Letters 28(8), 743 (2007)

5. J. Bizindavyi, A.S. Verhulst, Q. Smets, D. Verreck, B. Sorée, G. Groeseneken, Band-tails tunneling resolving the theory-experiment discrepancy in esaki diodes, IEEE Journal of the Electron Devices Society 6, 633 (2018)

6. U.E. Avci, R. Rios, K.J. Kuhn, I.A. Young, Comparison of power and performance for the tfet and mosfet and considerations for p-tfet pp. 869-872 (2011)

7. U.E. Avci, D.H. Morris, I.A. Young, Tunnel field-effect transistors: Prospects and challenges, IEEE Journal of the Electron Devices Society 3(3), 88 (2015)

8. K. Nigam, D. Sharma, et al., Approach for ambipolar behaviour suppression in tunnel fet by workfunction engineering, Micro \& Nano Letters 11(8), 460 (2016)

9. K. Boucart, A.M. Ionescu, Double-gate tunnel fet with high-k gate dielectric, IEEE transactions on electron devices 54(7), 1725 (2007)

10. V. Vijayvargiya, S.K. Vishvakarma, Effect of drain doping profile on double-gate tunnel field-effect transistor and its influence on device rf performance, IEEE Transactions on Nanotechnology 13(5), 974 (2014)

11. D.S. Yadav, D. Sharma, D.G. Sharma, S. Bajpai, High frequency analysis of gaasp/insb hetero-junction double gate tunnel field effect transistor pp. 1-6 (2018)

12. S. Kumar, D.S. Yadav, S. Saraswat, N. Parmar, R. Sharma, A. Kumar, A novel step-channel tfet for better subthreshold swing and improved analog/rf characteristics pp. 1-6 (2020)

13. M. Pala, D. Esseni, F. Conzatti, Impact of interface traps on the iv curves of inas tunnel-fets and mosfets: A full quantum study pp. 6-6 (2012)

14. C.K. Pandey, A. Singh, S. Chaudhury, Analysis of interface trap charges on dielectric pocket soi-tfet pp. 337-340 (2019)

15. Y. Qiu, R. Wang, Q. Huang, R. Huang, A comparative study on the impacts of interface traps on tunneling fet and mosfet, IEEE Transactions on Electron Devices 61(5), 1284 (2014)

16. J. Madan, R. Chaujar, Interfacial charge analysis of heterogeneous gate dielectric-gate all around-tunnel fet for improved device reliability, IEEE Transactions on Device and Materials Reliability 16(2), 227 (2016)

17. B.V. Chandan, K. Nigam, D. Sharma, S. Pandey, Impact of interface trap charges on dopingless tunnel fet for enhancement of linearity characteristics, Applied Physics A 124(7), 503 (2018)

18. T. Nepusz, A. Petróczi, L. Négyessy, F. Bazsó, A novel concept for field-effect transistors-the tunneling carbon nanotube fet $\mathbf{1}, 153$ (2005)

19. N.D. Chien, C.H. Shih, Oxide thickness-dependent effects of source doping profile on the performance of single-and double-gate tunnel field-effect transistors, Superlattices and Microstructures 102, 284 (2017)

20. K. Boucart, A.M. Ionescu, Length scaling of the double gate tunnel fet with a high-k gate dielectric, Solid-State Electronics 51(11-12), 1500 (2007)

21. H.B. Joseph, S.K. Singh, R. Hariharan, Y. Tarauni, D.J. Thiruvadigal, Simulation study of gated nanowire inas/si hetero p channel tfet and effects of interface trap, Materials Science in Semiconductor Processing 103, 104605 (2019)

22. D.A. Buchanan, Scaling the gate dielectric: Materials, integration, and reliability, IBM Journal of Research and Development 43(3), 245 (1999) 
23. B.R. Raad, D. Sharma, P. Kondekar, K. Nigam, D.S. Yadav, (IEEE, 2016), vol. 63, pp. 3950-3957

24. R. Ranjan, K. Pradhan, P. Sahu, et al., A comprehensive investigation of silicon film thickness $(t \mathrm{si})$ of nanoscale $\mathrm{dg}$ tfet for low power applications, Advances in Natural Sciences: Nanoscience and Nanotechnology 7(3), 035009 (2016)

25. D.S. Yadav, D. Sharma, S. Tirkey, D.G. Sharma, S. Bajpai, D. Soni, S. Yadav, M. Aslam, N. Sharma, Heteromaterial cptfet with high-frequency and linearity analysis for ultra-low power applications, Micro \& Nano Letters 13(11), 1609 (2018)

26. S.M. Sze, Semiconductor devices: physics and technology (John wiley \& sons, 2008)

27. S. Chander, S.K. Sinha, S. Kumar, P.K. Singh, K. Baral, K. Singh, S. Jit, Temperature analysis of ge/si heterojunction soi-tunnel fet, Superlattices and Microstructures 110, $162(2017)$

28. D.S. Yadav, D. Sharma, S. Tirkey, V. Bajaj, A systematic investigation of the integrated effects of gate underlapping, dual work functionality and hetero gate dielectric for improved performance of cp tfets, Journal of Computational Electronics 17(1), 118 (2018)

29. A. Kranti, G.A. Armstrong, Nonclassical channel design in mosfets for improving ota gain-bandwidth trade-off, IEEE Transactions on Circuits and Systems I: Regular Papers 57(12), 3048 (2010)

30. M. Aslam, D. Sharma, D. Soni, S. Yadav, B.R. Raad, D.S. Yadav, N. Sharma, Effective design technique for improvement of electrostatics behaviour of dopingless tfet: proposal, investigation and optimisation, Micro \& Nano Letters 13(10), 1480 (2018)

31. J. Madan, R. Chaujar, Interfacial charge analysis of heterogeneous gate dielectric-gate all around-tunnel fet for improved device reliability, IEEE Transactions on Device and Materials Reliability 16(2), 227 (2016)

32. P.K. Verma, S.K. Gupta, Proposal of charge plasma based recessed source/drain dopingless junctionless transistor and its linearity distortion analysis for circuit applications, Silicon pp. 1-28 (2020) 
Figures

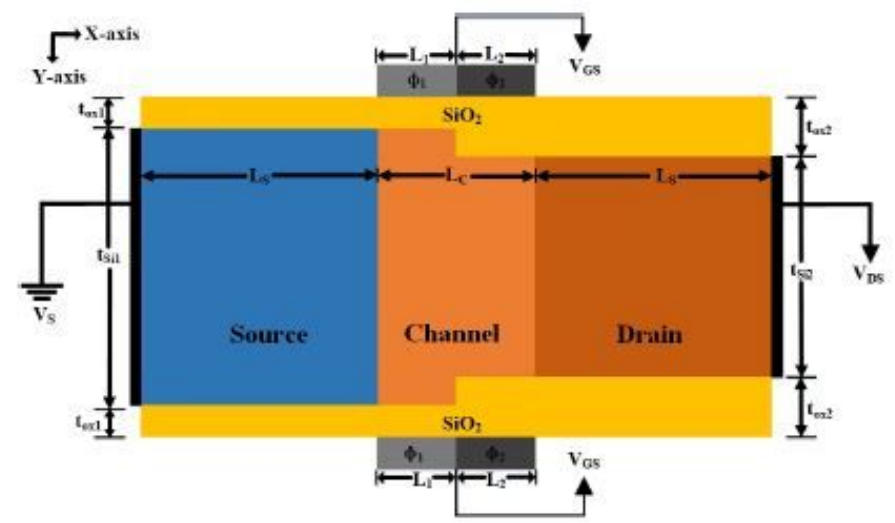

(a)

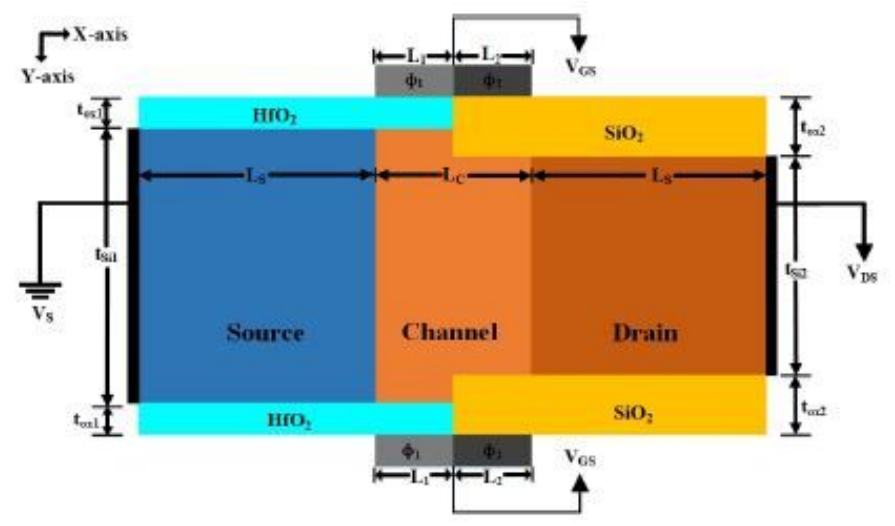

(b)

Figure 1

Device Schematic view of (a) DMG SC-TEFT (b) HD DMG SC-TEFT 


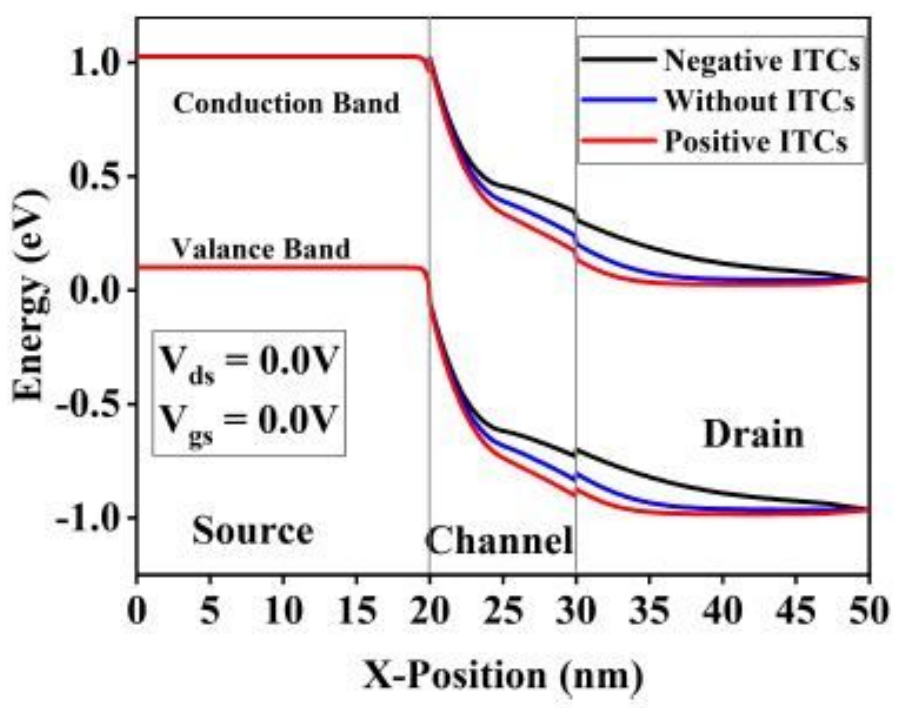

(a)

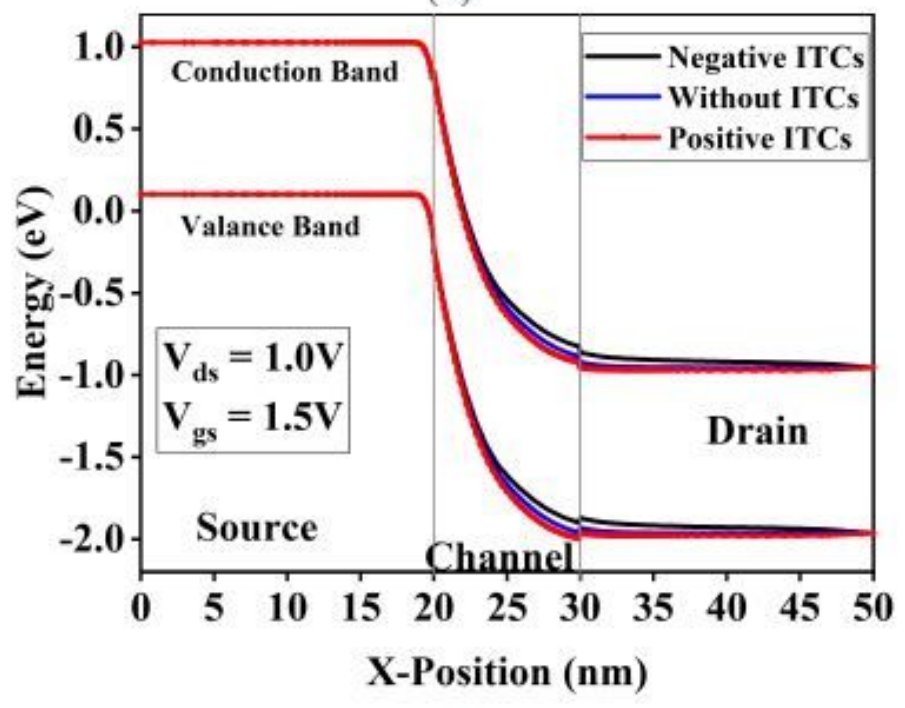

(c)

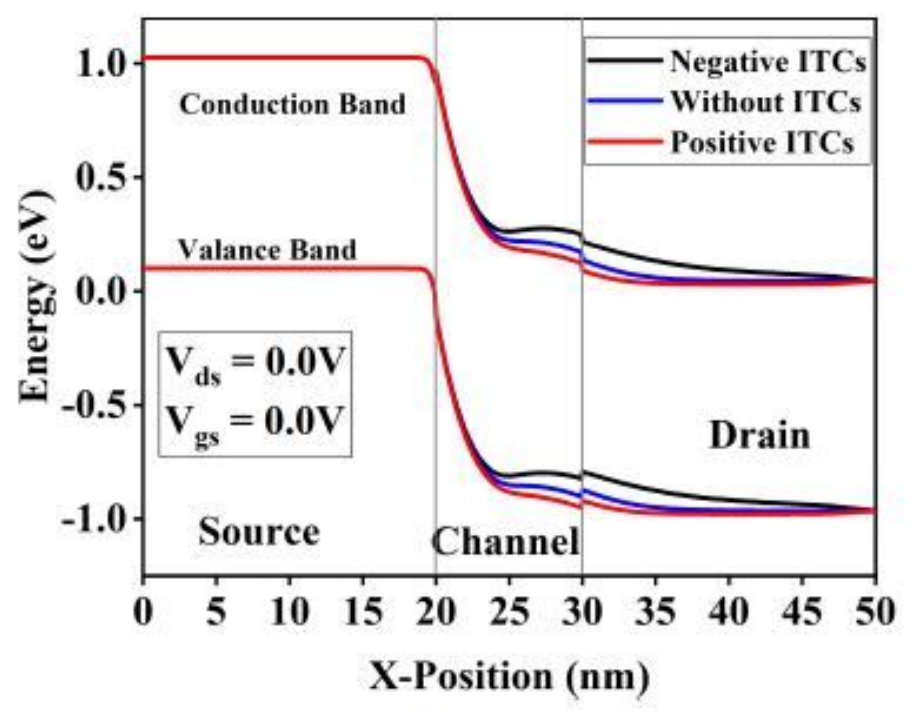

(b)

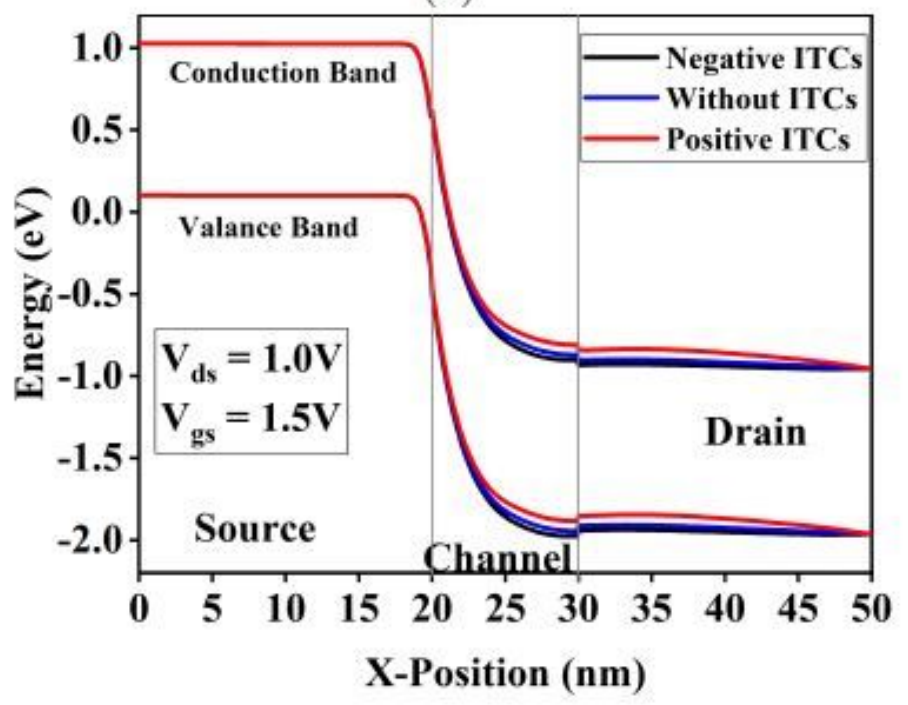

(d)

Figure 2

Impact of ITC on (a) Energy Band of DMG SC-TFET under Thermal Equilibrium (b) Energy Band of HD DMG SC-TFET under Thermal Equilibrium (c) Energy Band of DMG SC-TFET under ON-State (d) Energy Band of HD DMG SC-TFET under ON-State 


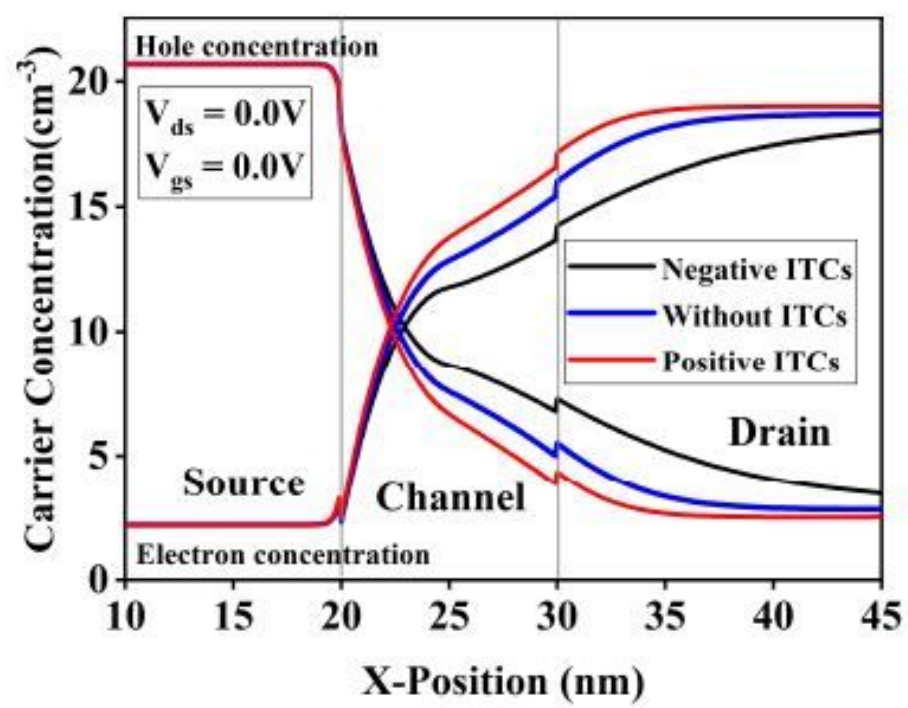

(a)

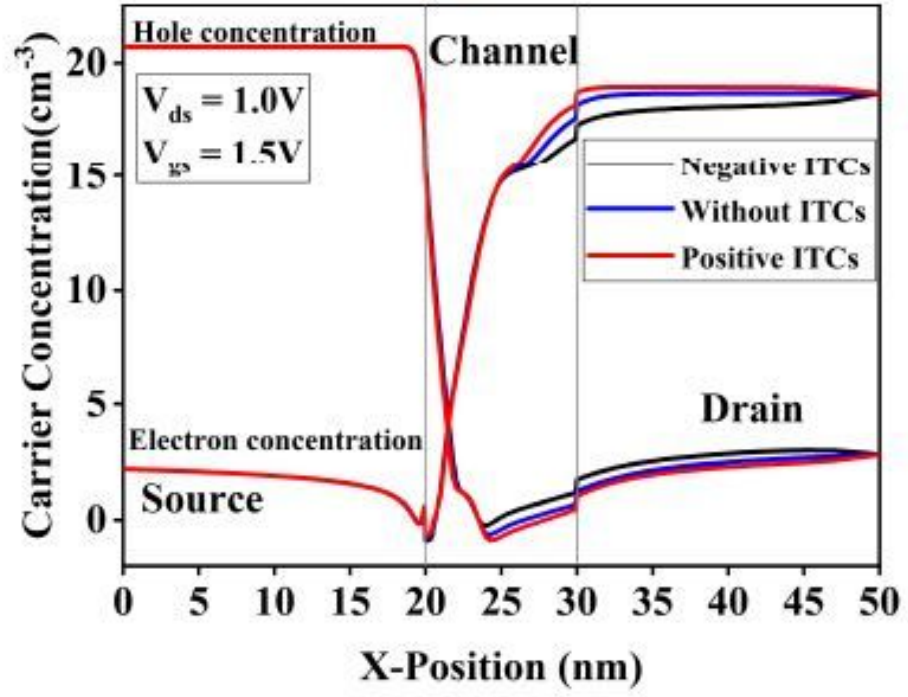

(c)

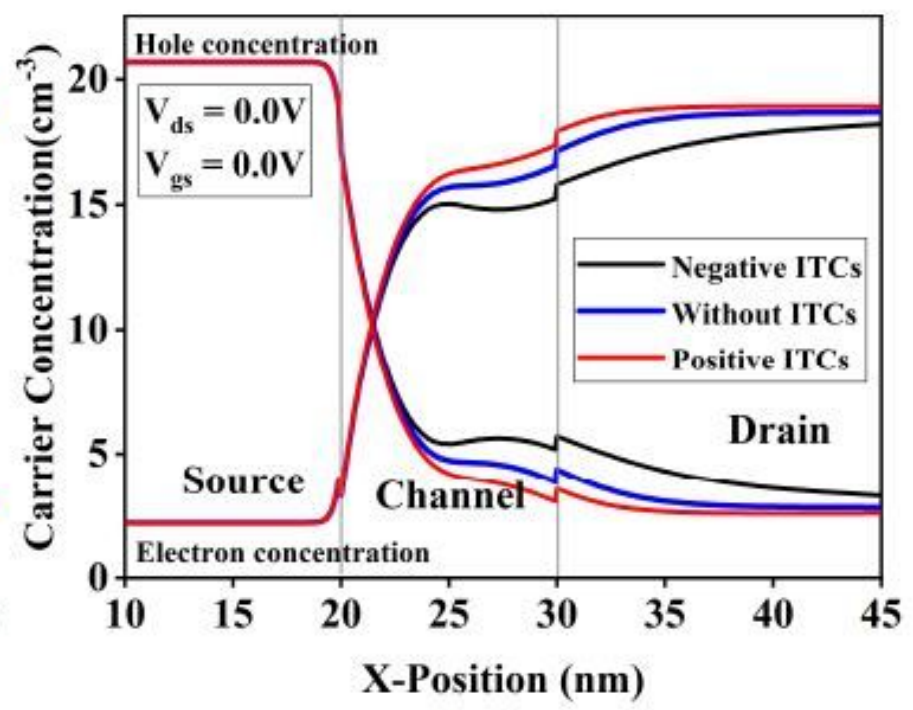

(b)

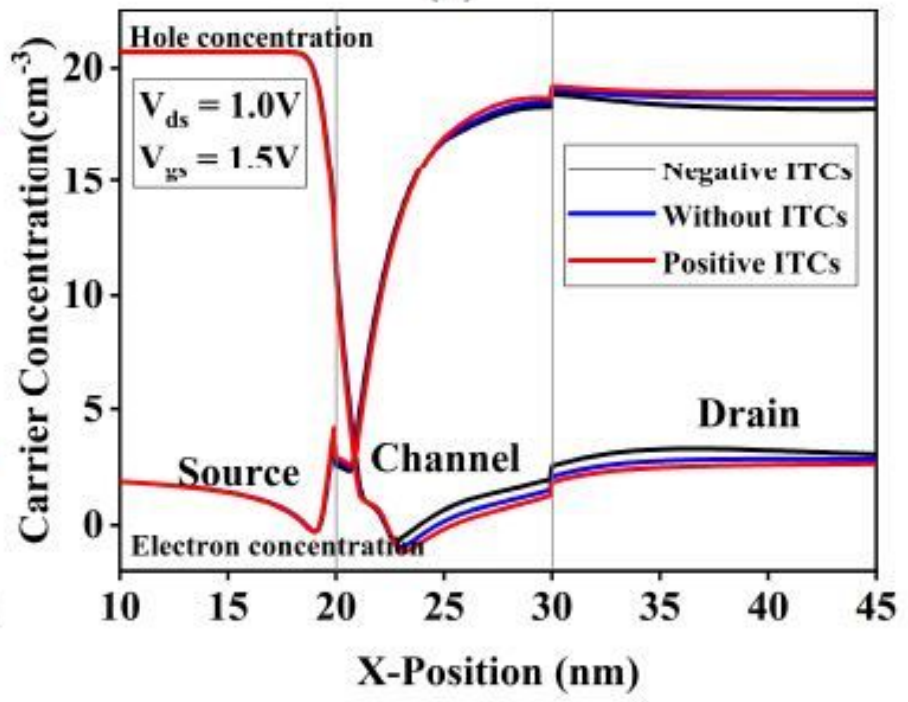

(d)

Figure 3

Impact of ITC on (a) Carrier Concentration of DMG SC-TFET under Thermal Equilibrium (b) Carrier Concentration of HD DMG SC-TFET under Thermal Equilibrium (c) Carrier Concentration of DMG SC-TFET under ON-State (d) Carrier Concentration of HD DMG SC-TFET under ON-State 


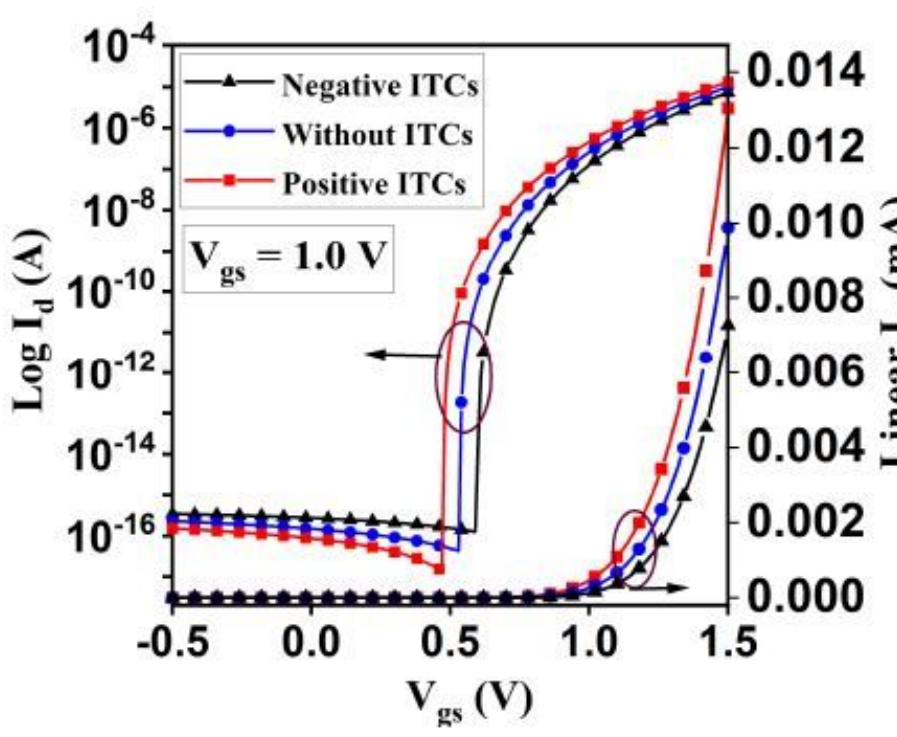

(a)

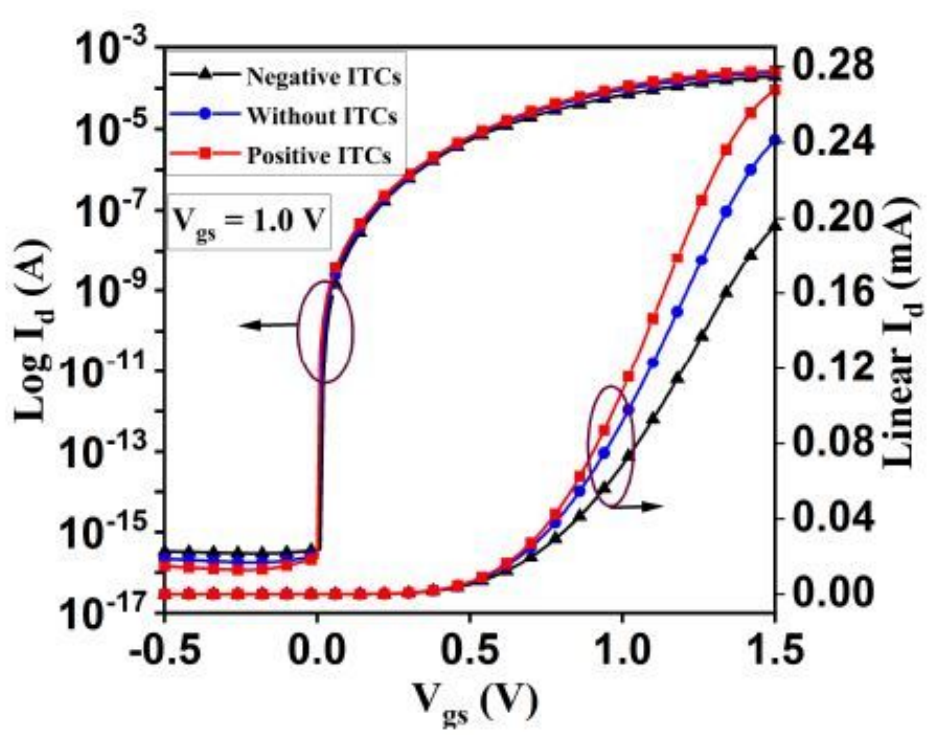

(b)

Figure 4

Impact of ITCs on transfer characteristics (a) DMG SC-TEFT (b) HD DMG SC-TEFT

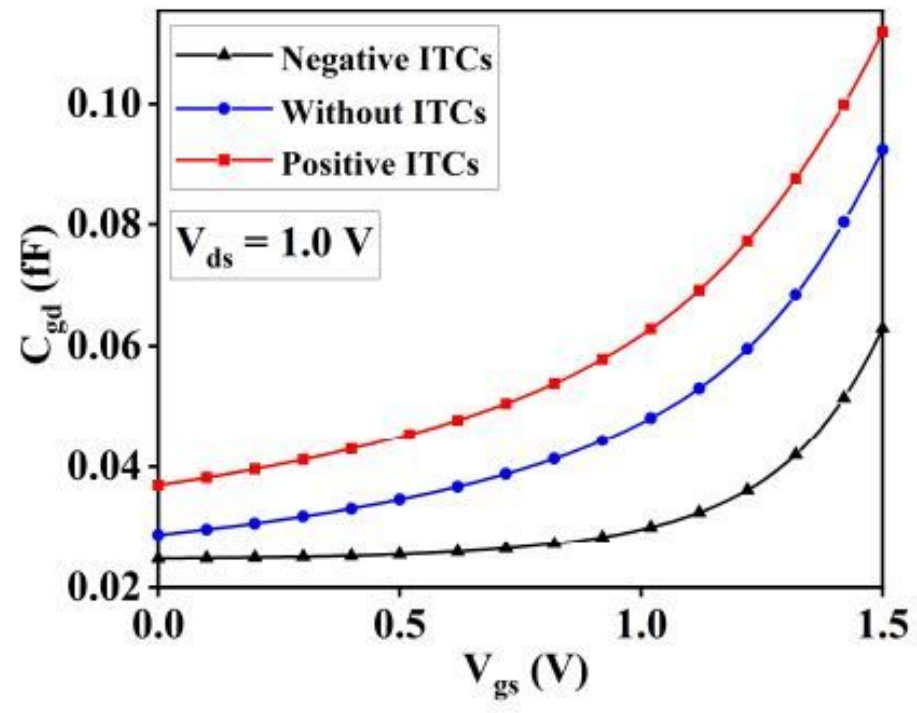

(a)

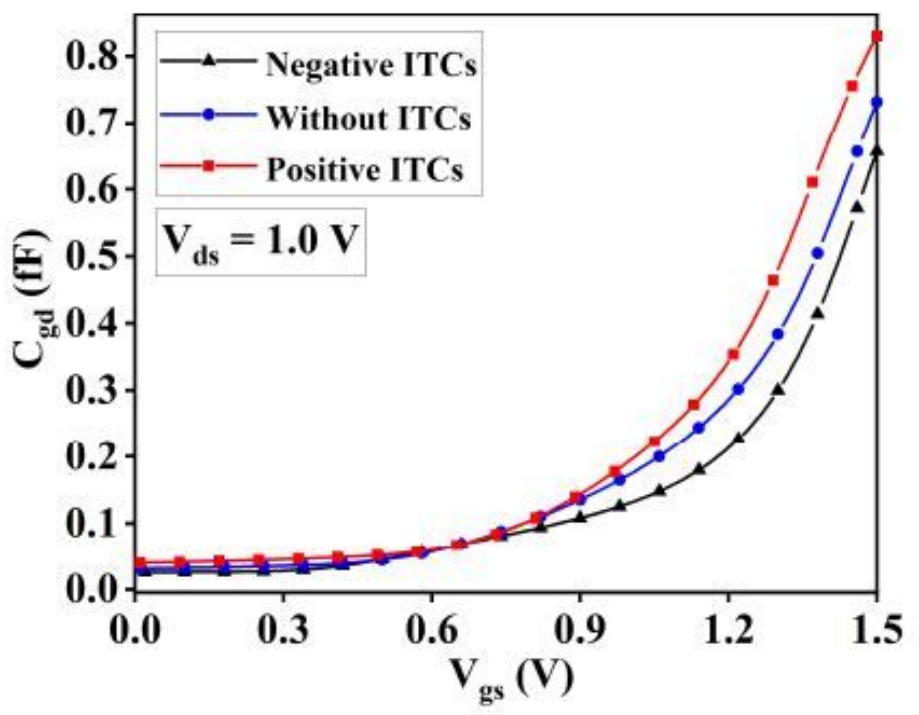

(b)

Figure 5

Impact of ITCs on Cgd (a) DMG SC-TEFT (b) HD DMG SC-TEFT 


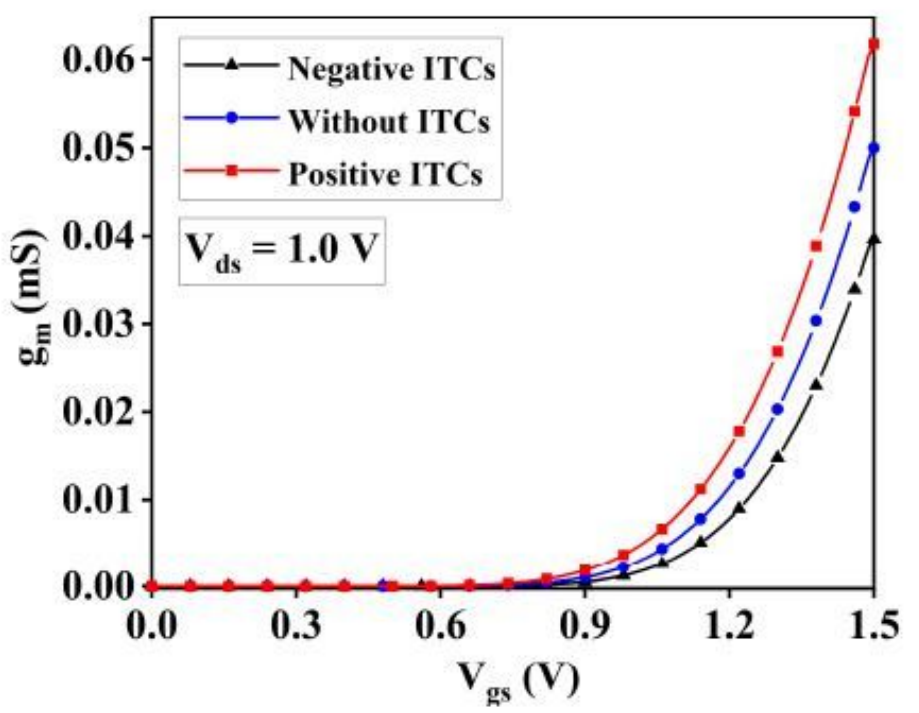

(a)

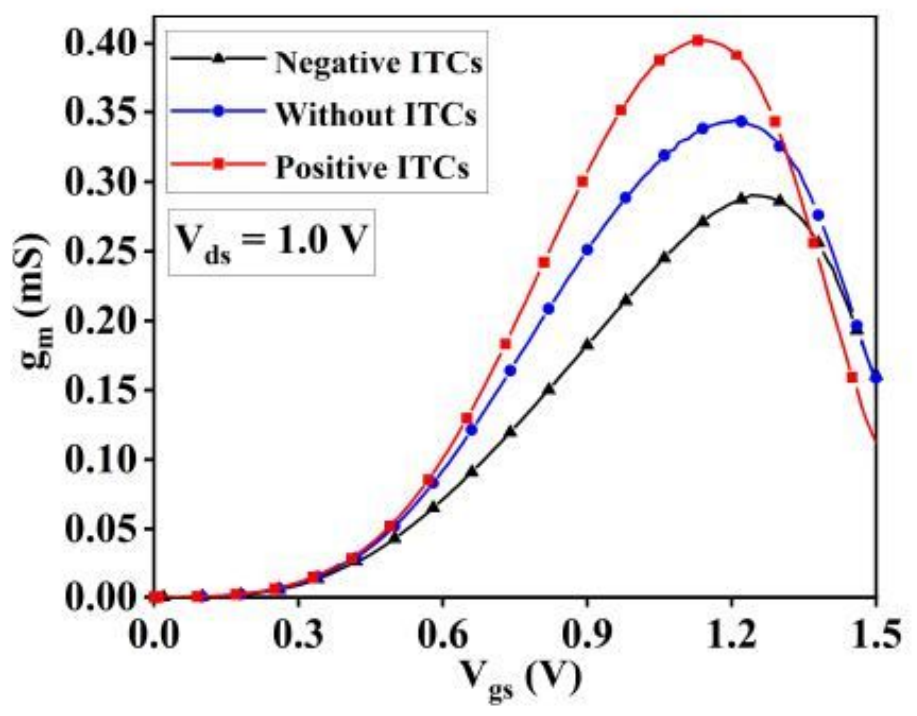

(b)

Figure 6

Impact of ITCs on gm (a) DMG SC-TEFT (b) HD DMG SC-TEFT

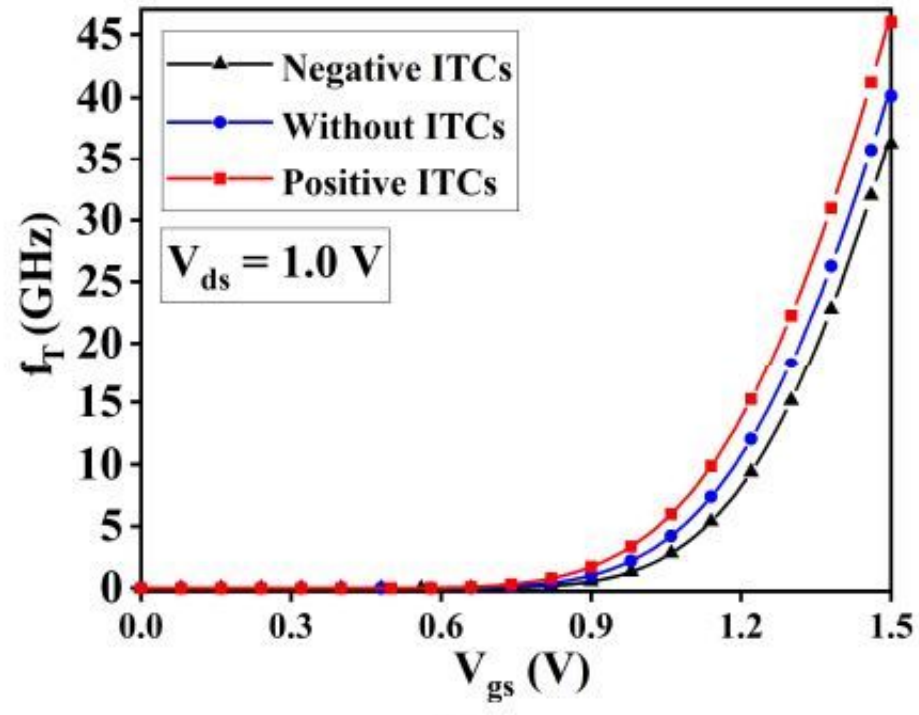

(a)

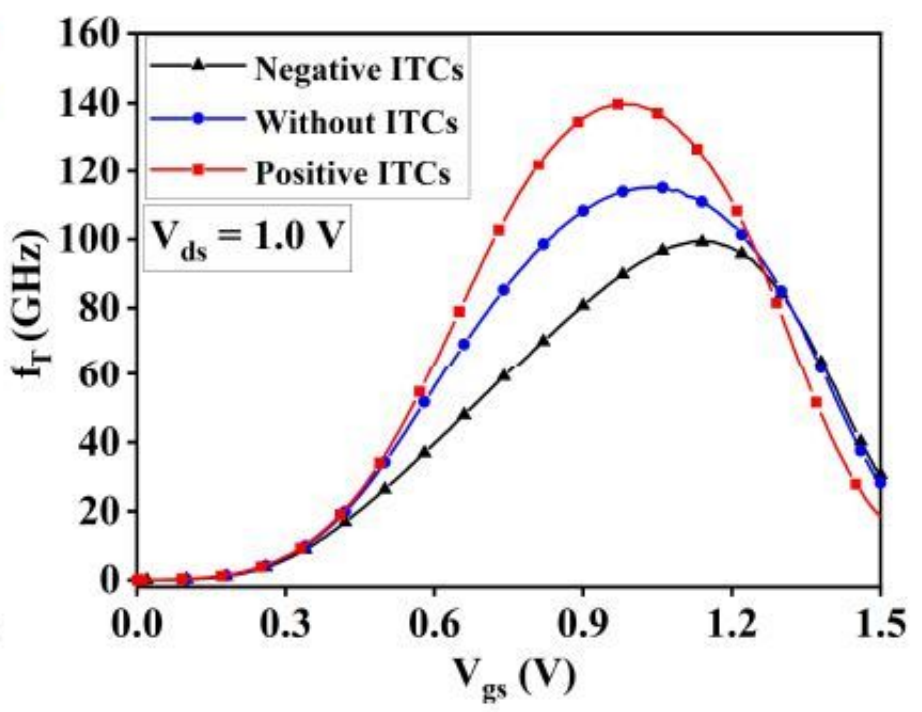

(b)

Figure 7

Impact of ITCs on fT (a) DMG SC-TEFT (b) HD DMG SC-TEFT 


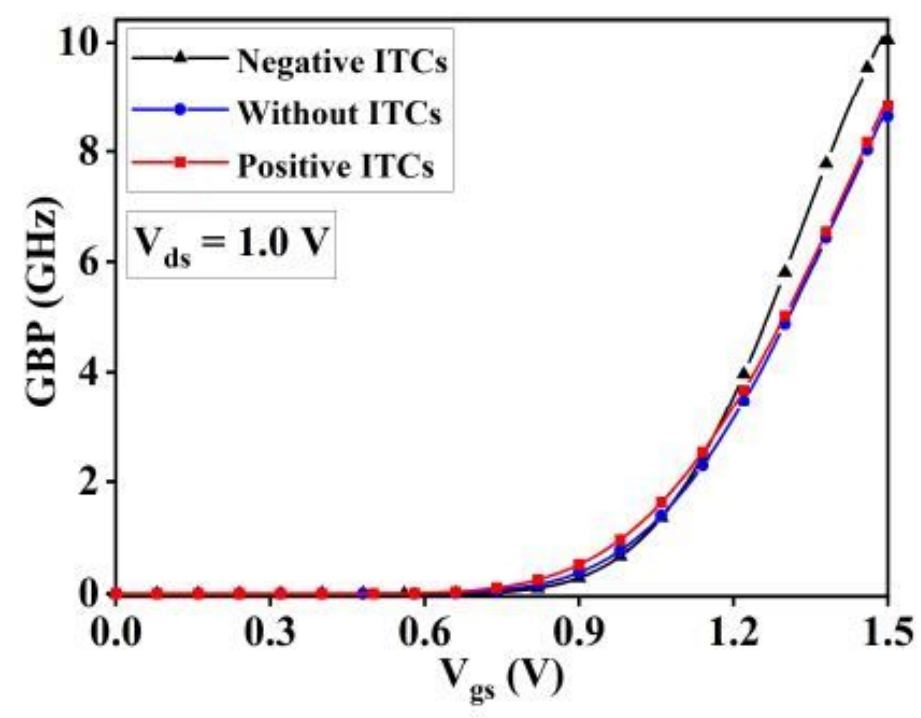

(a)

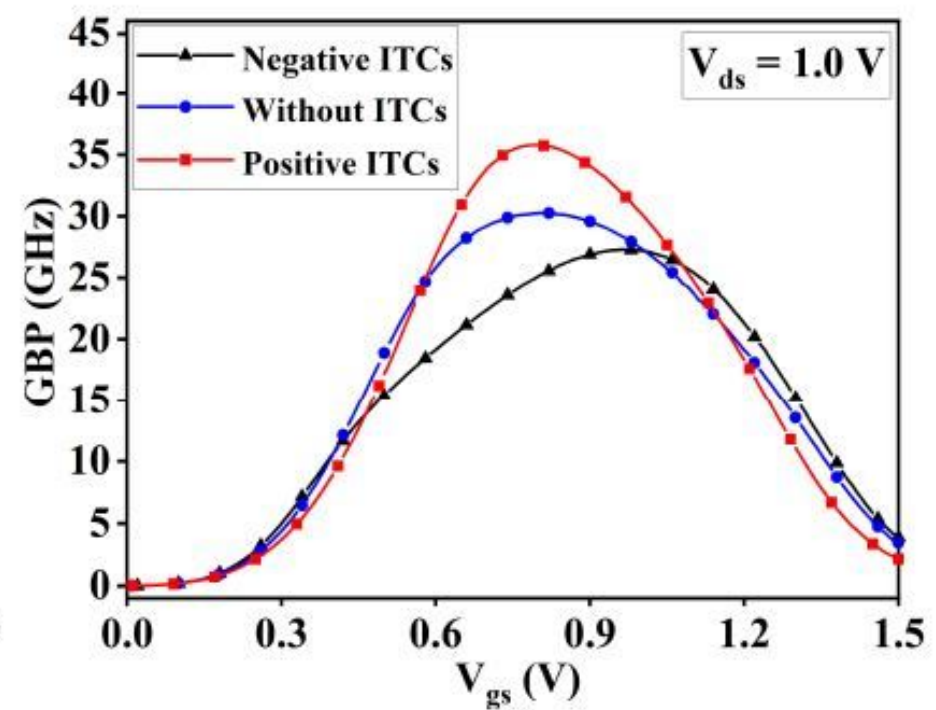

(b)

Figure 8

Impact of ITCs on GBP (a) DMG SC-TEFT (b) HD DMG SC-TEFT

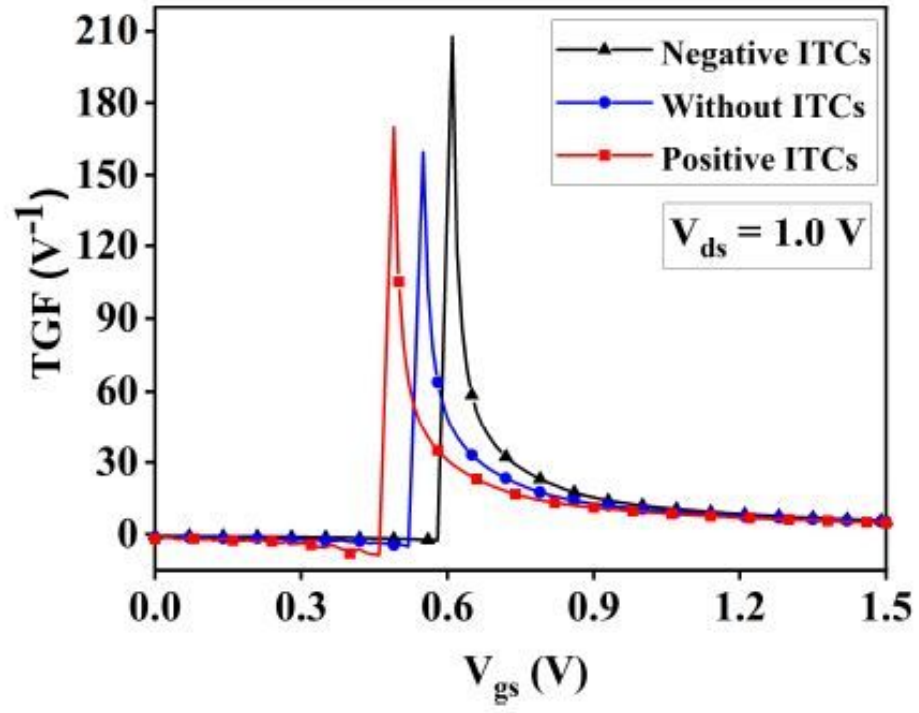

(a)

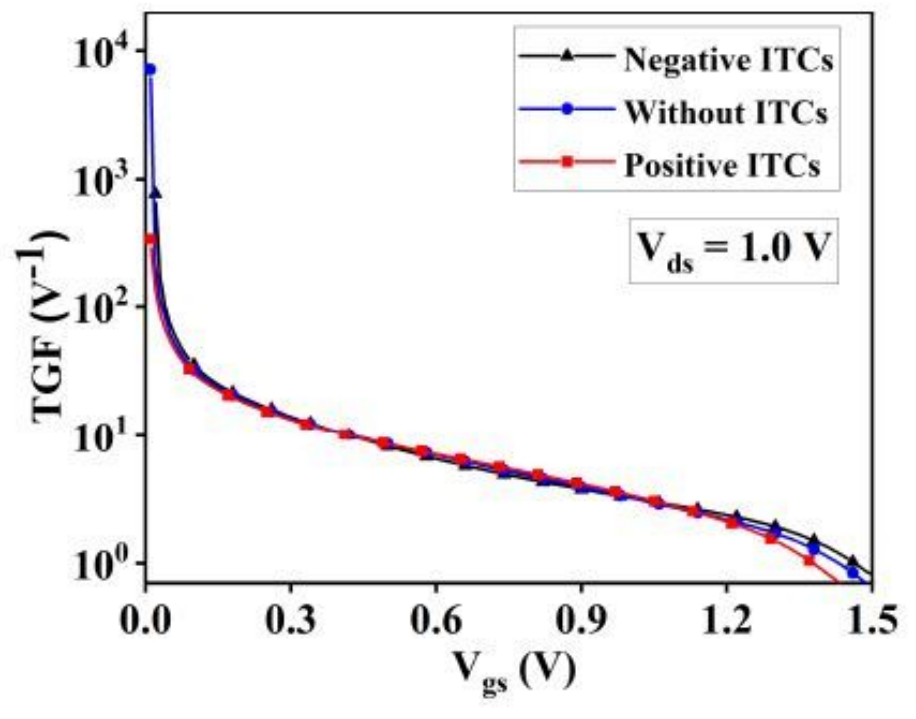

(b)

Figure 9

Impact of ITCs on TGF (a) DMG SC-TEFT (b) HD DMG SC-TEFT 


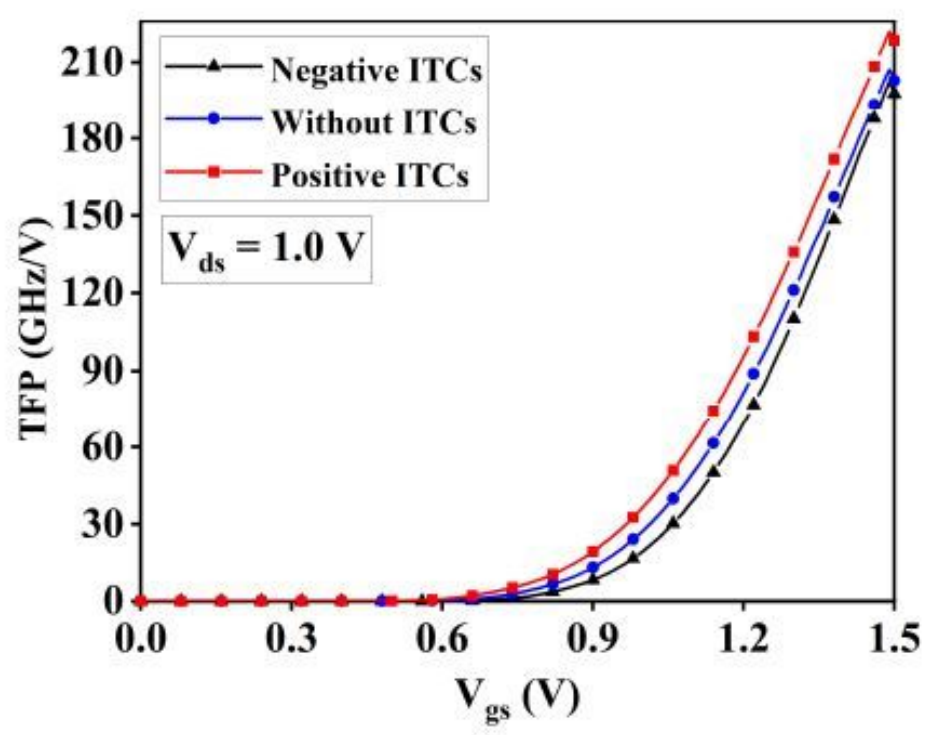

(a)

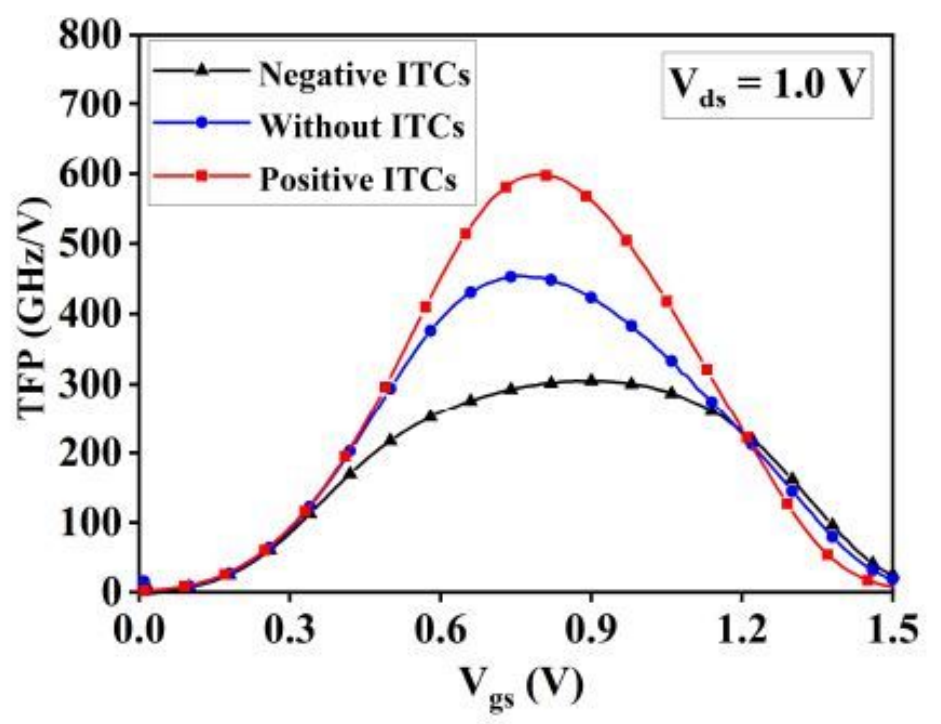

(b)

Figure 10

Impact of ITCs on TFP (a) DMG SC-TEFT (b) HD DMG SC-TEFT

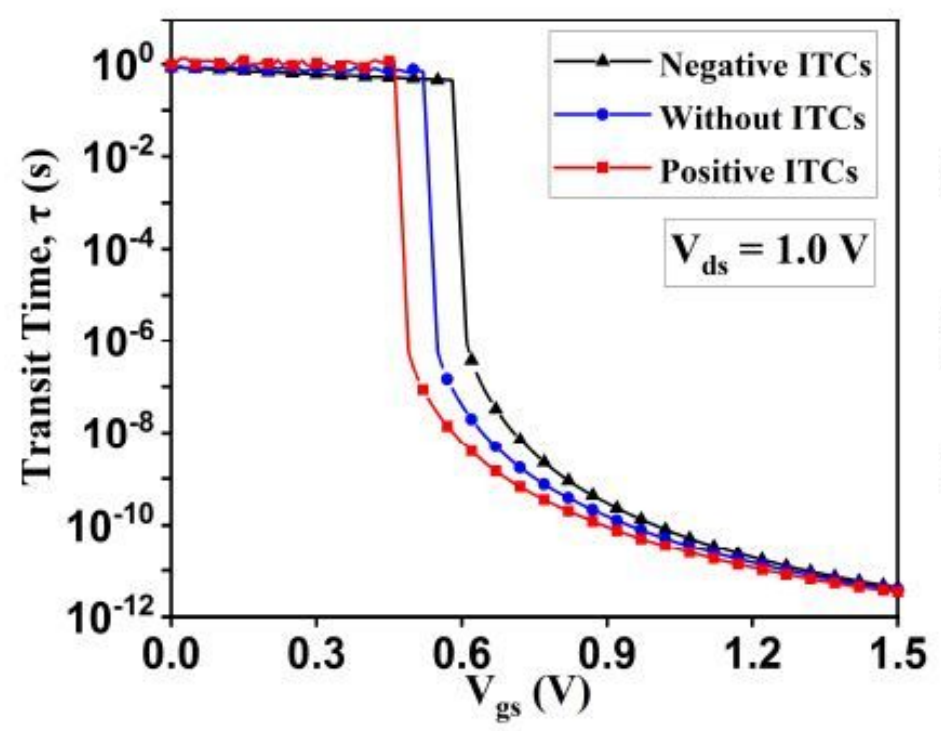

(a)

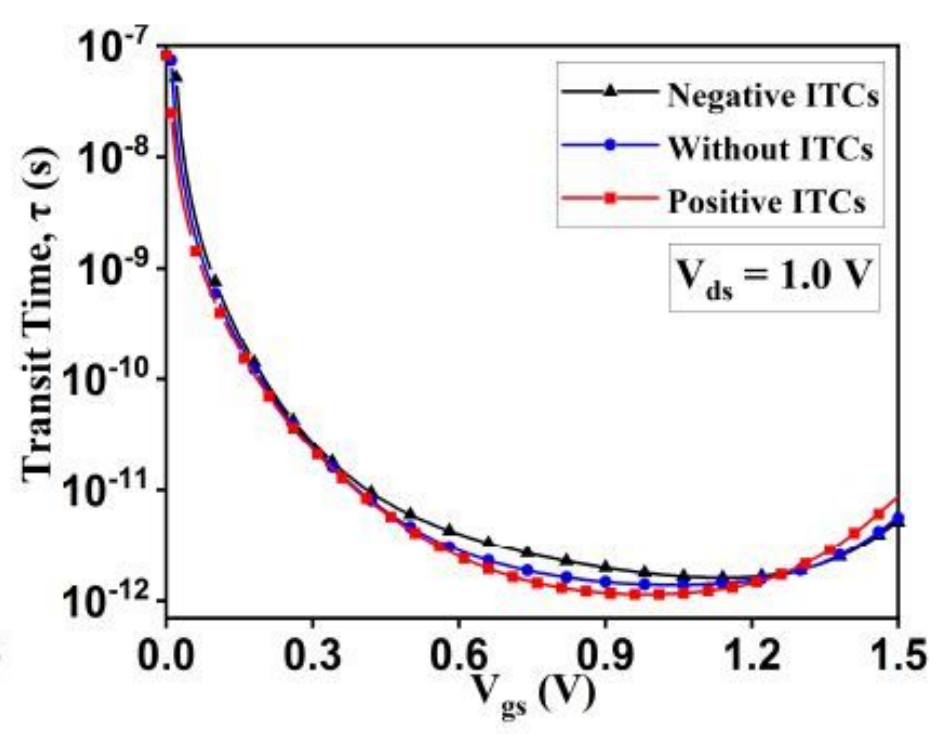

(b)

Figure 11

please see the manuscript file for the full caption 

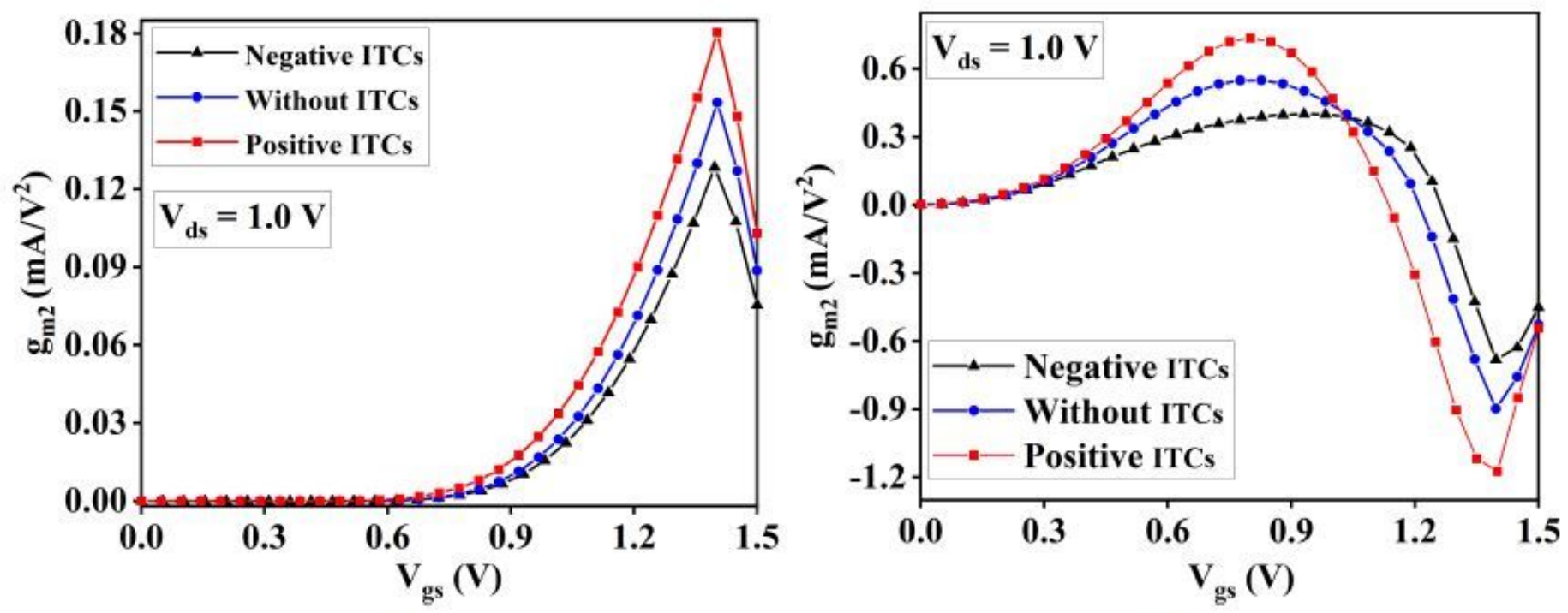

(a)

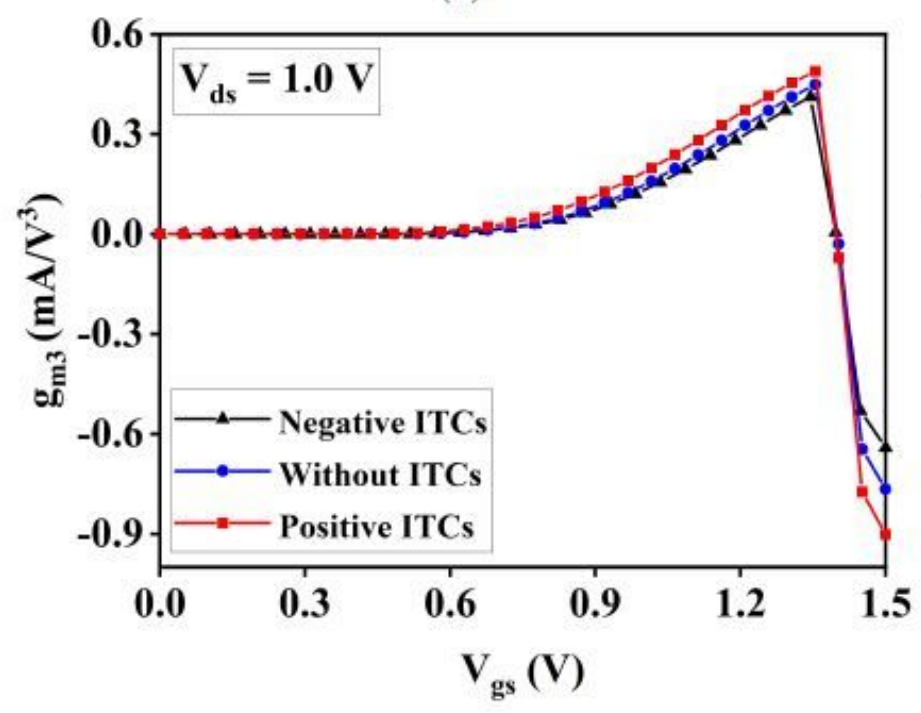

(b)

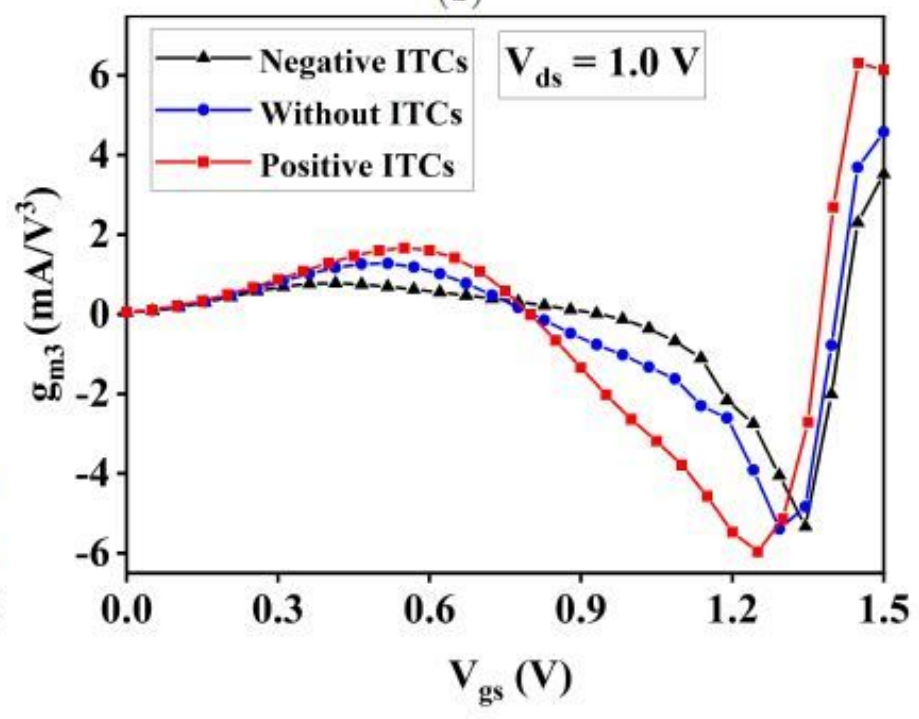

(c)

(d)

Figure 12

Impact of ITCs on (a) gm2 of DMG SC-TEFT (b) gm2 of HD DMG SC-TEFT (c) gm3 of DMG SC-TEFT (d) gm3 of HD DMG SC-TEFT 


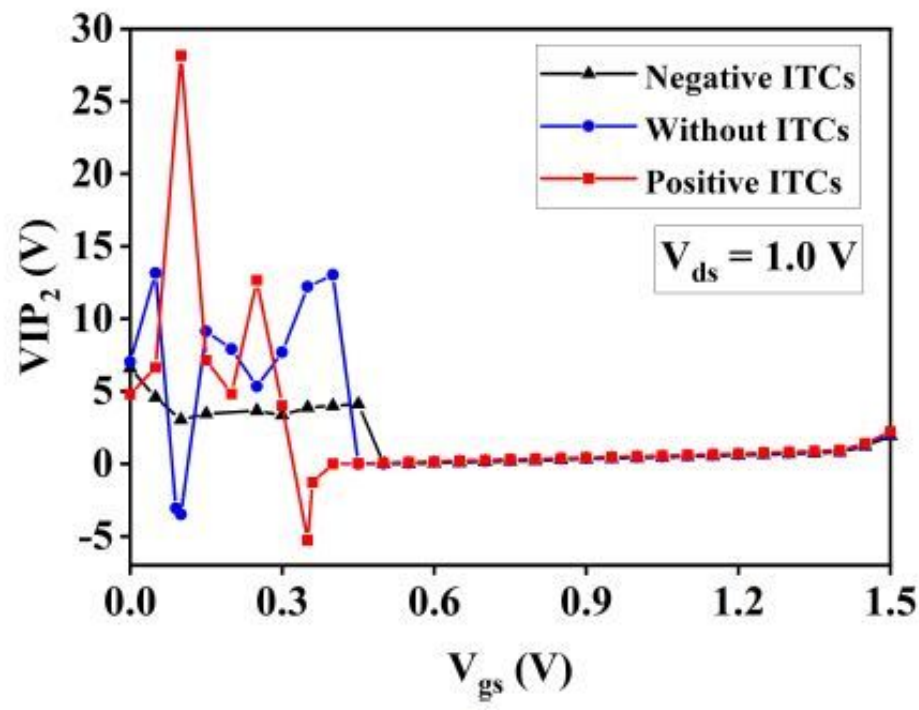

(a)

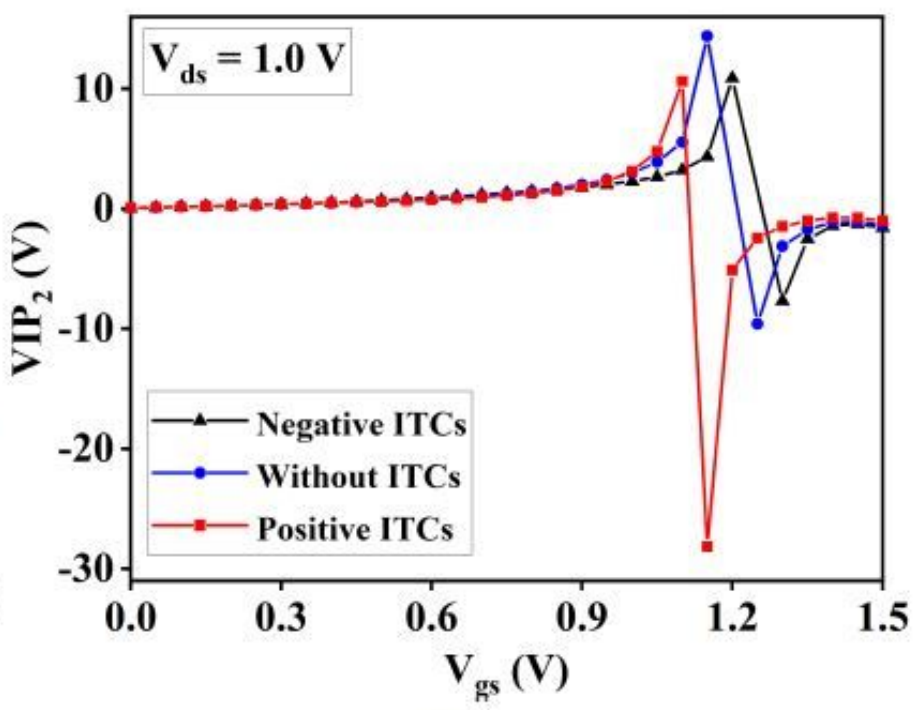

(b)

Figure 13

Impact of ITCs on VIP2 (a) DMG SC-TEFT (b) HD DMG SC-TEFT

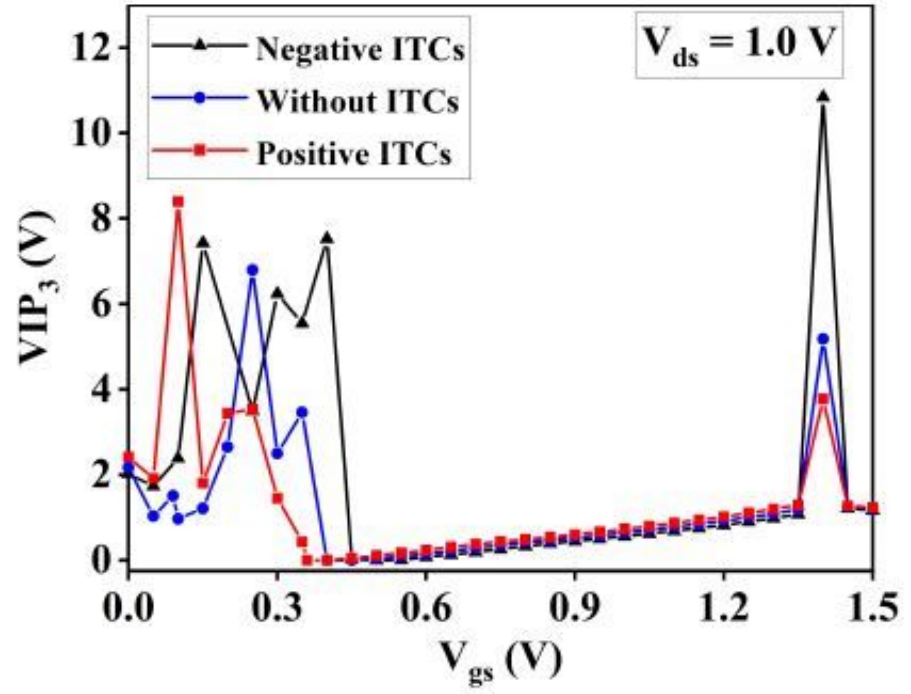

(a)

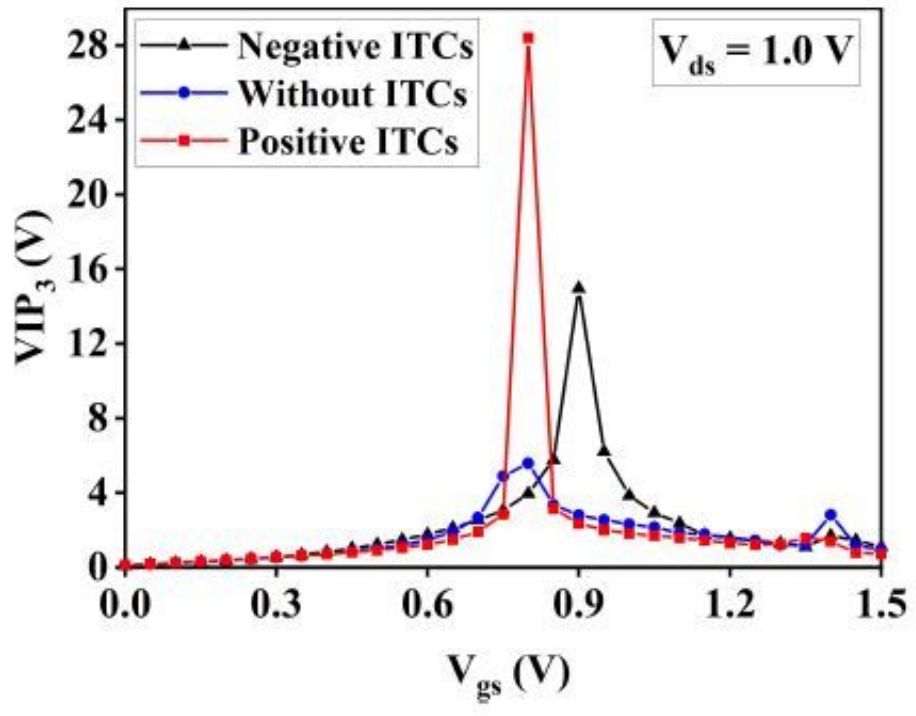

(b)

Figure 14

Impact of ITCs on VIP3 (a) DMG SC-TEFT (b) HD DMG SC-TEFT 


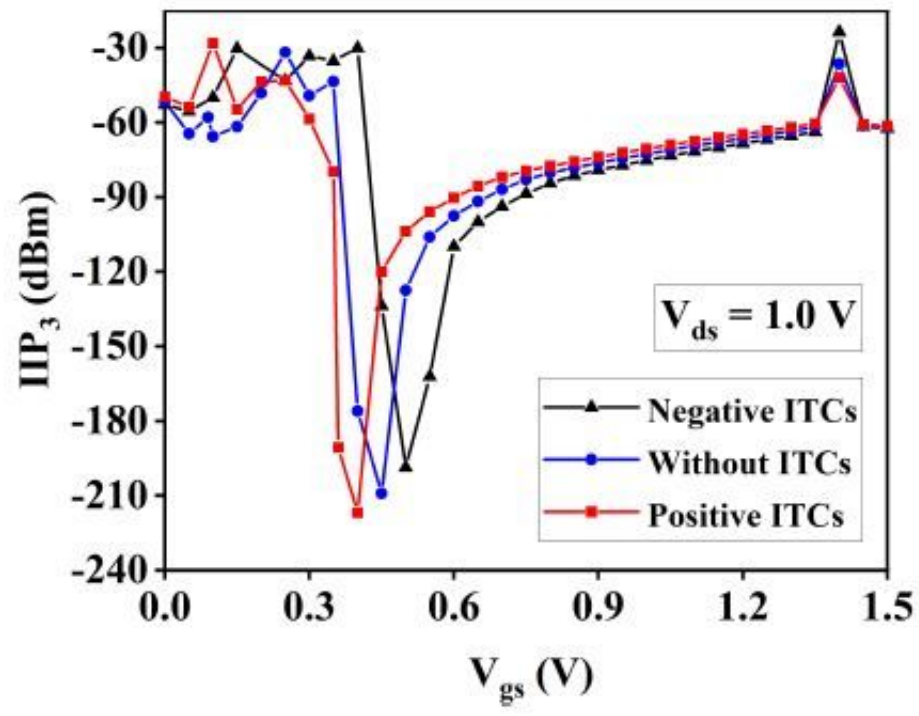

(a)

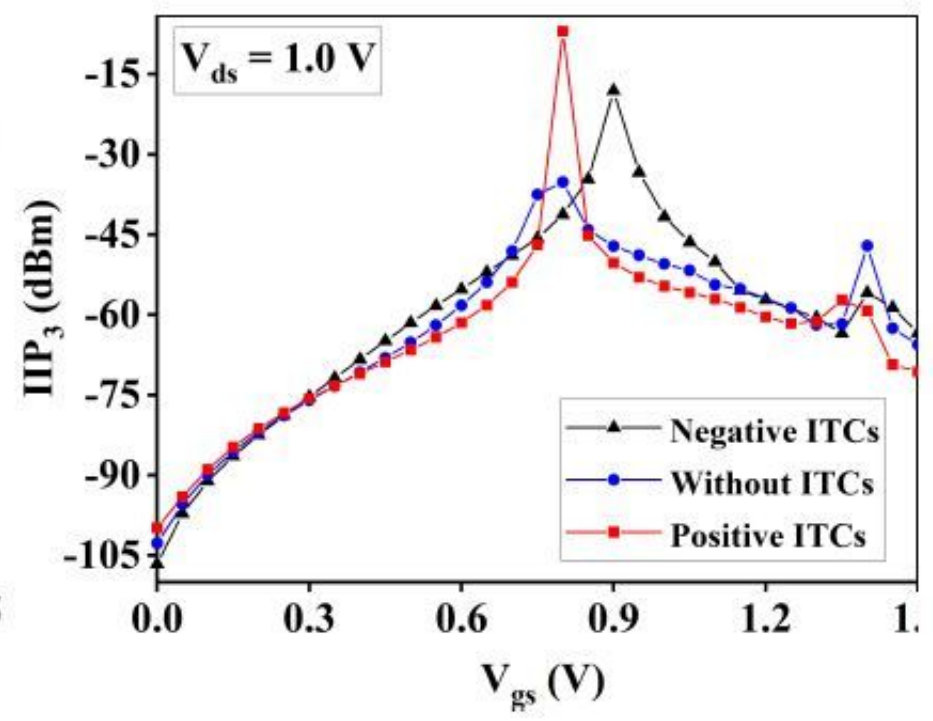

(b)

Figure 15

Impact of ITCs on IIP3 (a) DMG SC-TEFT (b) HD DMG SC-TEFT

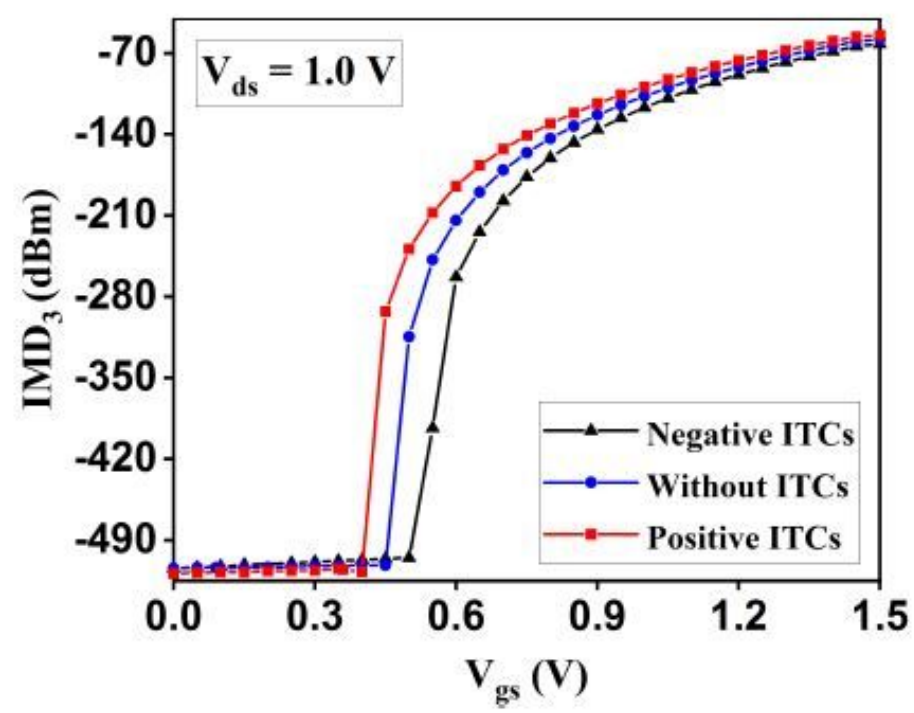

(a)

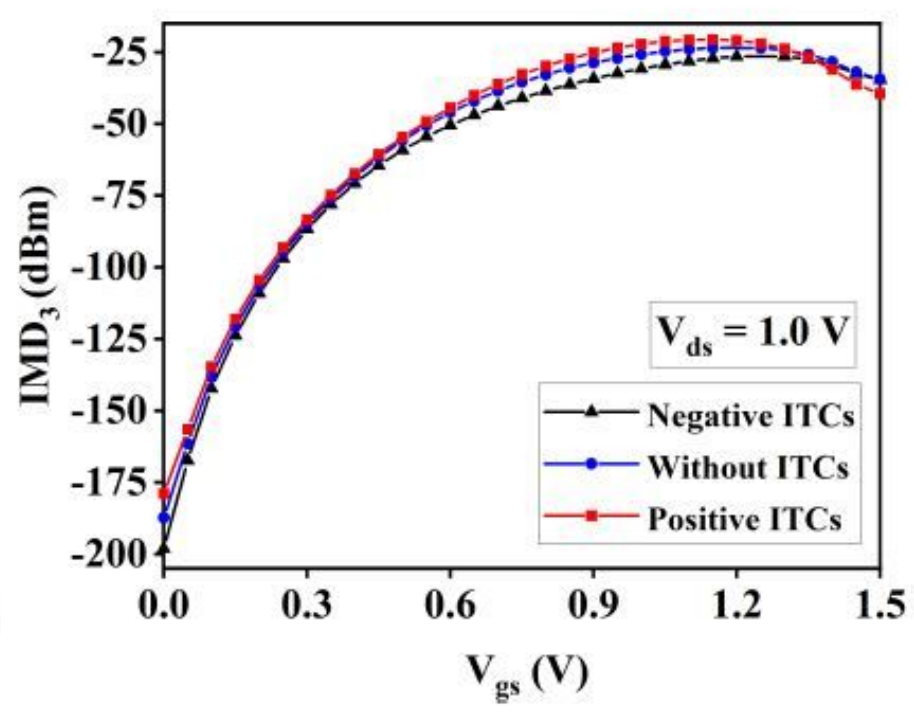

(b)

Figure 16

Impact of ITCs on IMD3 (a) DMG SC-TEFT (b) HD DMG SC-TEFT 


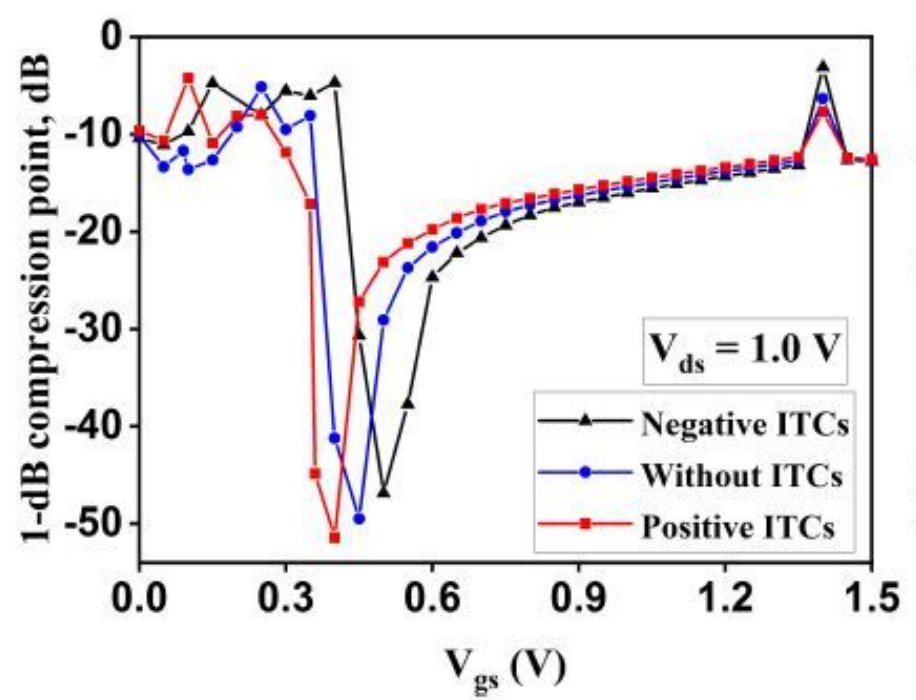

(a)

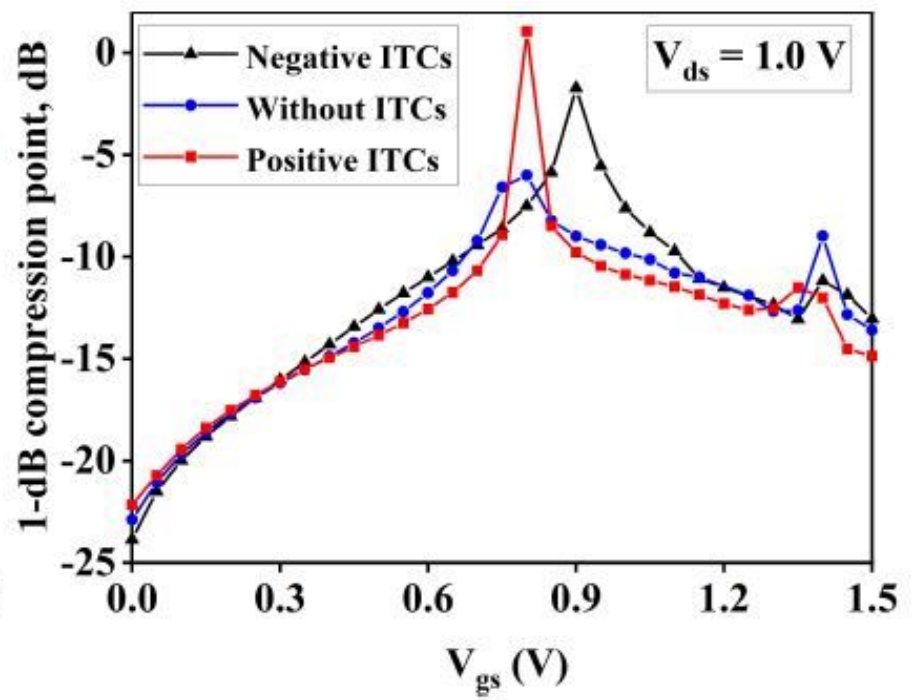

(b)

Figure 17

Impact of ITCs on 1dB compression point (a) DMG SC-TEFT (b) HD DMG SC-TEFT 